\title{
A stabilized finite element approximation of low speed thermally coupled flows
}

\author{
Javier Principe and Ramon Codina \\ Departament de Resistència de Materials i Estructures a l'Enginyeria, \\ Universitat Politècnica de Catalunya, Barcelona, Spain
}

Finite element approximation

835

Received 2 April 2007 Revised 7 September 2007 Accepted 20 September 2007

\begin{abstract}
Purpose - The purpose of this paper is to describe a finite element formulation to approximate thermally coupled flows using both the Boussinesq and the low Mach number models with particular emphasis on the numerical implementation of the algorithm developed.

Design/methodology/approach - The formulation, that allows us to consider convection dominated problems using equal order interpolation for all the valuables of the problem, is based on the subgrid scale concept. The full Newton linearization strategy gives rise to monolithic treatment of the coupling of variables whereas some fixed point schemes permit the segregated treatment of velocity-pressure and temperature. A relaxation scheme based on the Armijo rule has also been developed.

Findings - A full Newtown linearization turns out to be very efficient for steady-state problems and very robust when it is combined with a line search strategy. A segregated treatment of velocity-pressure and temperature happens to be more appropriate for transient problems.

Research limitations/implications - A fractional step scheme, splitting also momentum and continuity equations, could be further analysed.

Practical implications - The results presented in the paper are useful to decide the solution strategy for a given problem.

Originality/value - The numerical implementation of a stabilized finite element approximation of thermally coupled flows is described. The implementation algorithm is developed considering several possibilities for the solution of the discrete nonlinear problem.
\end{abstract}

Keywords Finite element analysis, Stability (control theory), Flow

Paper type Research paper

\section{Introduction}

The general description of a fluid flow involves the solution of the compressible Navier Stokes equations. It is widely accepted that these equations provide an accurate description of any problem in fluid mechanics. This set of equations, the mathematical formulation of the physical principles of mass, momentum and energy conservation coupled with a state equation, is very complex and very little is known about its mathematical structure. Partial results on the boundary conditions that make the problem well posed, on the existence of a solution and on uniqueness can be found in (Lions, 1996). The mathematical complexity of the problem is the manifestation of the also complex physical behavior of flows. Many different nonlinear physical mechanisms are coupled in fluid mechanics problems. For these reasons, depending on the physics of the problem under consideration, different models can be derived from the compressible Navier Stokes equations (Lions, 1996; Zeytounian, 1990).

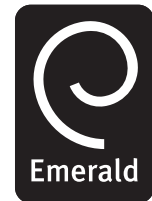

International Journal of Numerical Methods for Heat \& Fluid Flow Vol. 18 No. $7 / 8,2008$ pp. $835-867$

(C) Emerald Group Publishing Limited 0961-5539 
HFF

$18,7 / 8$

\section{6}

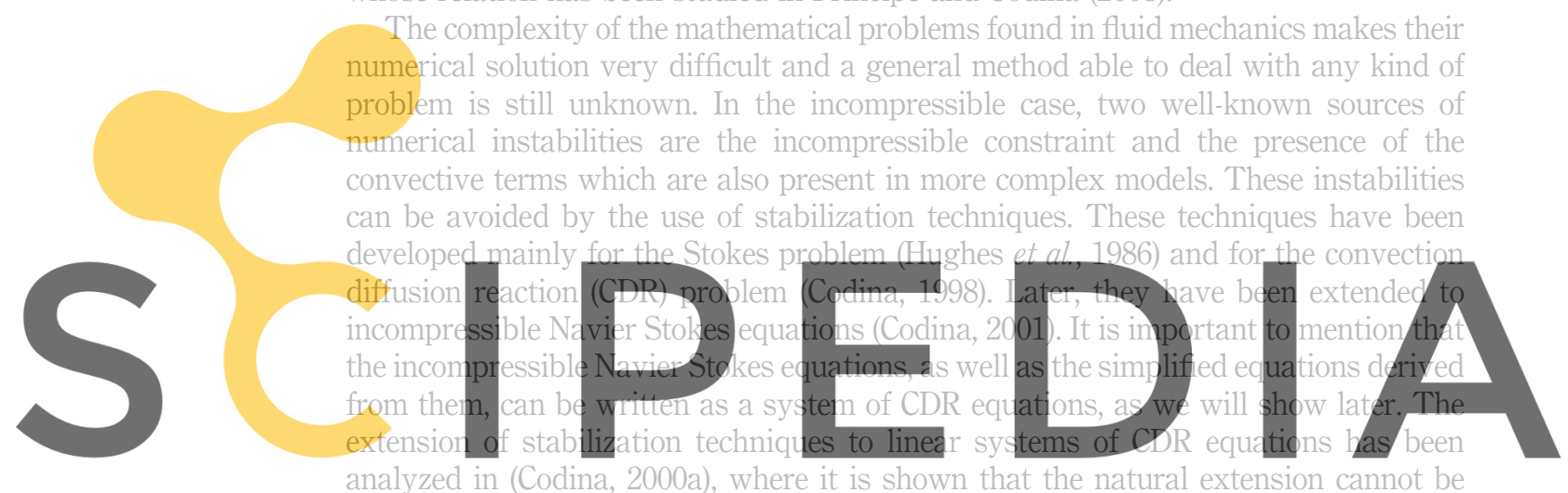

The derivation of these reduced sets of equations is based on some assumptions on the problem, usually made in terms of some dimensionless parameters that measure the relative importance of different physical processes, like the Mach or Reynolds numbers. The most important of these models is described by the incompressible Navier Stokes equations. This set of equations is smaller than the compressible one and its mathematical structure is much better understood. Furthermore, two physical effects that are difficult to predict, shock waves and sound waves, are not found in incompressible problems. However, many important flows cannot be considered as incompressible due to the presence of thermal effects. In such kind of problems, another class of simplified equations can be derived: the Boussinesq equations and the low Mach number equations. Section 2 is devoted to the presentation of such models whose relation has been studied in Principe and Codina (2008).

Register for free at httpspyw w. scipedia.com to downlead the version without the watipermark parameters is still unknown. These techniques have also been extended to transient nonlinear problems in Codina (2002), Codina et al. (2007) and Codina and Principe (2007), where a nonlinear time dependent stabilization was developed. It has also been realized that these techniques could be used as a physical model to take nonlinear-phenomena into account. In particular, thermal turbulence, which is usually taken into account through the introduction of a turbulent Prandtl number whose physical meaning and adequate value are not well understood, would be consider in a very natural way. Section 3 is devoted to the presentation of a stabilized finite element formulation of the Boussinesq equations and the low Mach number equations.

However, it is neither our purpose to focus on the derivation of the physical models considered nor on the development of a nonlinear stabilization, but on numerical implementation aspects. Apart from the potential numerical instabilities, another manifestation of the complexity of the problems considered is their highly nonlinear nature. Therefore, their numerical solution requires a proper linearization strategy which can be written, in general, as a fixed point scheme as shown in Section 4. We test the proposed approach in Section 5, where numerical examples illustrating the accuracy and performance of the scheme are shown. Some conclusions are drawn in Section 7. 


\section{Physical models}

The flow of a compressible fluid in a domain $\Omega$ is described in terms of the velocity $(u)$, pressure $(p)$, density $(\rho)$, and temperature $(\vartheta)$ fields, which arc solutions of (Batchelor, 1967; Lions, 1996):

$$
\begin{aligned}
\frac{D \rho}{D t}+\rho \nabla \cdot u & =0 \\
\rho \frac{D u}{D t}+\nabla_{p} & =\nabla \cdot\left(2 \mu \varepsilon^{\prime}(u)\right)+\rho g \\
\rho c_{p} \frac{D \vartheta}{D t}-\beta \vartheta \frac{D p}{D t} & =\nabla \cdot(k \nabla \vartheta)+\Phi+Q \\
\rho & =\mathrm{F}(p, \vartheta)
\end{aligned}
$$

Finite element approximation

where $(\mathbf{D} / \mathbf{D} t)=(\partial / \partial t)+u \cdot \nabla$ is the material derivative, $g$ the external source of momentum, $Q$ the external source of energy, $\varepsilon^{\prime}(u)=\varepsilon(u)-(1 / 3) \nabla \cdot u$, I the deviatoric part of the rate of deformation tensor $(\varepsilon$ is the symmetric part of the velocity gradient, $\left.\varepsilon(u)=(1 / 2) \nabla u+\nabla u^{t}\right), \mu$, the kinematic viscosity, $c_{p}$ the constant pressure specific heat, $k$ the thermal conductivity, $\beta$ the thermal expansion coefficient and $\Phi$ (the Rayleigh dissipation function) is a non-negative contribution due to mechanical

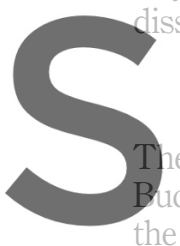
issipation of energy in
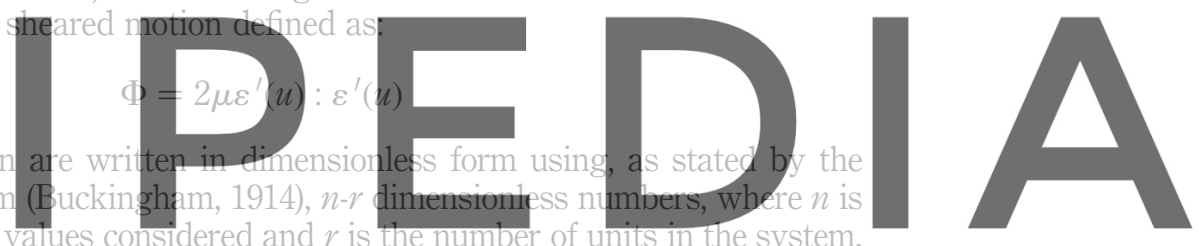

In this case $r=4$ (length, time, mass, and temperature units) and we consider reference

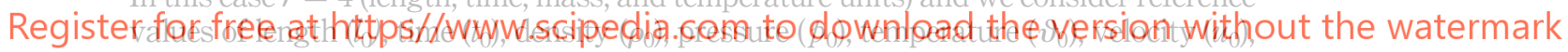
viscosity $\left(u_{0}\right)$, conductivity $\left(k_{0}\right)$, specific heat $\left(c_{p 0}\right)$, external acceleration $\left(g_{0}\right)$ and energy (Q0) that permit us to define dimensionless variables (denoted by ${ }^{\sim}$ ) as:

$$
\begin{gathered}
x=l_{0} \tilde{x}, \quad t=t_{0} \tilde{t}, \quad \rho=\rho_{0} \tilde{\rho}, \quad p=p_{0} \tilde{p}, \quad \vartheta=\vartheta_{0} \tilde{\vartheta} \\
u=u_{0} \tilde{u}, \quad g=g_{0} \tilde{g}, \quad Q=Q_{0} \tilde{Q}, \quad \mu=\mu_{0} \tilde{\mu}, \quad k=k_{0} \tilde{k}, \quad c_{p}=c_{p 0} \tilde{c}_{p}
\end{gathered}
$$

The choice of viscosity and conductivity reference values is needed to allow variable physical properties (temperature dependent, for example). We assume that $\rho_{0}, p_{0}, \vartheta_{0}$ are related by the state equation, so we have ten reference values, giving rise to the six dimensionless numbers: Strouhal, Mach, Reynolds, Peclet, Froude and a heat release rate number, defined as:

$$
\begin{gathered}
\mathbf{S}=\frac{l_{0}}{u_{0} t_{0}}, \quad \mathbf{M}=\frac{u_{0}}{\sqrt{p_{0} / \rho_{0}}}, \quad \mathbf{R}=\frac{\rho_{0} u_{0} l_{0}}{\mu_{0}} \\
P=\frac{\rho_{0} c_{p_{0}} u_{0} l_{0}}{k_{0}}, \quad \mathbf{F}=\frac{u_{0}}{\sqrt{g_{0} l_{0}}}, \quad H=\frac{t_{0} Q_{0}}{\rho_{0} c_{p_{0}} \vartheta_{0}}
\end{gathered}
$$

The dimensionless equations are (omitting $\sim$ ): 


\section{$\mathrm{HFF}$}

$18,7 / 8$

\section{8}

$$
\begin{gathered}
\mathbf{S} \frac{\partial \rho}{\partial t}+\nabla \cdot(\rho u)=0 \\
\rho\left(\mathbf{S} \frac{\partial u}{\partial t}+u \cdot \nabla u\right)+\frac{1}{\mathbf{M}^{2}} \nabla_{p}=\frac{1}{\mathbf{R}} \nabla \cdot\left(2 \mu \varepsilon^{\prime}(u)\right)+\frac{1}{\mathbf{F}^{2}} \rho g \\
\rho c_{p}\left(\mathbf{S} \frac{\partial \vartheta}{\partial t}+u \cdot \nabla \vartheta\right)-\mathbf{S}_{t} \beta \vartheta\left(\mathbf{S} \frac{\partial p}{\partial t}+u \cdot \nabla_{p}\right)=\frac{\mathbf{M}^{2}}{\mathbf{R}} \Phi+\frac{1}{P} \nabla \cdot(k \nabla \vartheta)+H S Q
\end{gathered}
$$

where $\mathbf{S}_{t}$ is a parameter that depends on the state equation and is given by:

$$
\mathbf{S}_{t}=\frac{p_{0}}{\rho_{0} c_{p_{0}} \vartheta_{0}}
$$

The simplified models that we consider are obtained taking an appropriate limit of the dimensionless numbers. The first model, the low Mach number approximation, has been studied first in Rehm and Baum (1978) in the inviscid case, and generalized to the viscous case in Paolucci (1982). A rigorous derivation including combustion was presented in Majda and Sethian (1985) and its relation to the Boussinesq approximation was studied in Principe and Codina (2008). The limit of $\mathbf{M} \rightarrow 0$ is found using standard procedures of asymptotic analysis described for example in

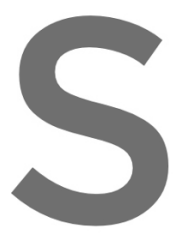

Keyorkian and Cole (1981). The firs
of the small paraneter considered:
\[ \xi(x, t, \mathbf{M})=\xi(\vartheta(x, t) \]
for $\xi=\nu, \boldsymbol{\xi}=p, \xi=\rho, \xi=\vartheta$. Th
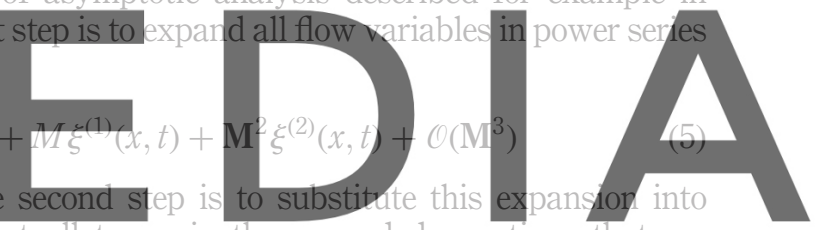

equations (2)-(4) and to require that all terms in the expanded equations that are

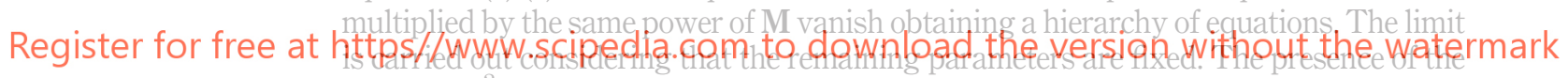
factor $\mathrm{ML}^{-2}$ in the momentum equation leads to:

$$
\nabla p^{(0)}=0
$$

from where $p^{(0)}=p^{(0)}(t)$ and the following set of equations is obtained:

$$
\begin{aligned}
& \mathbf{S} \frac{\mathrm{d} p^{(0)}}{\mathrm{d} t}+\nabla \cdot\left(\rho^{(0)} u^{(0)}\right)=0 \\
& \rho^{(0)}\left(\mathbf{S} \frac{\partial u^{(0)}}{\mathrm{d} t}+u^{(0)} \cdot \nabla u^{(0)}\right)+\nabla p^{(2)}=\frac{1}{\mathbf{R}} \nabla \cdot\left(2 \mu^{(0)} \varepsilon^{\prime}\left(u^{(0)}\right)\right)+\frac{1}{\mathbf{F}^{2}} \rho^{(0)} g \\
& \rho^{(0)} c_{p}^{(0)}\left(\mathbf{S} \frac{\partial \vartheta^{(0)}}{\partial t}+u^{(0)} \cdot \nabla \boldsymbol{\vartheta}^{(0)}\right)-\mathbf{S}_{t} \beta \vartheta^{(0)} \mathbf{S} \frac{\mathrm{d} p^{(0)}}{\mathrm{d} t}
\end{aligned}
$$

which is closed with a state equation of the form:

$$
\rho^{(0)}=\mathbf{F}\left(p^{(0)}, \vartheta^{(0)}\right)
$$

As the only parameter present in the compressible equations is $\mathbf{M}^{2}$, first order fields vanish. Therefore, the pressure splits into two contributions: $p^{(0)}$, a reference thermodynamic pressure constant over the whole domain, and $p^{(2)}$, a mechanical 
pressure that is determined from a velocity constraint playing the same role as in incompressible equations. The reference thermodynamic pressure is determined by the external pressure when the domain is "open" to the atmosphere (Rehm and Baum, 1978; Principe and Codina, 2008) and is determined from a global balance determined from the continuity and energy equations using the state equation when the domain is closed (i.e. when velocities are prescribed over the whole boundary). In the case of an ideal gas, we have that:

$$
p^{(0)} \nabla \cdot u^{(0)}=-\frac{1}{\gamma} \mathbf{S} \frac{\mathrm{d} p^{(0)}}{\mathrm{d} t}+\frac{1}{P} \nabla \cdot\left(k^{(0)} \nabla \vartheta^{(0)}\right)+H S Q
$$

and integrating over the domain gives the ordinary differential equation:

$$
p^{(0)} \int_{\partial \Omega} u^{(0)} \cdot n=-\frac{V \Omega}{\gamma} \mathrm{S} \frac{\mathrm{d} p^{(0)}}{\mathrm{d} t}+\frac{1}{P} \int_{\partial \Omega} q^{(0)} \cdot n+H S \int_{\Omega} Q
$$

which is solved to determine the reference pressure.

The second model we will consider is the widely used Boussinesq (1903) approximation which was proposed in 1903, and consists in neglecting the variations of density except where they multiply the gravity acceleration. Several justifications of the Boussinesq approximation were proposed (Spiegel and Veronis, 1960; Mihaljan, 1962; Perez Cordon and Velarde, 1975; Gra and Giorgini, 1976), but asymptotic analyses were only perfo 8003). As shown in Princip

following the same proce hat $\mathrm{M}=\mathrm{F}^{2}$ (here and be sources are small, or more precisely, that $H \simeq \mathbb{M}$. In this case, the external force is

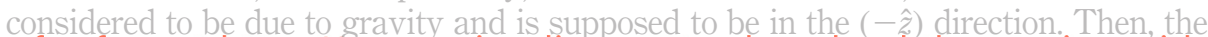

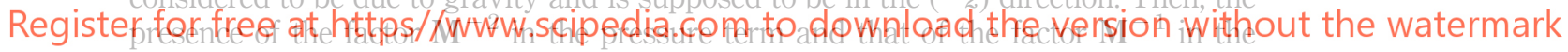
gravity term of the momentum equation lead to:

$$
\nabla p^{(0)}=0
$$

and:

$$
\nabla p^{(1)}=-\rho^{(0)} \hat{z}
$$

from where it follows that $p^{(0)}=p^{(0)}(t), p^{(1)}=p^{(1)}(z, t)$ and that $\rho^{(0)}=\rho^{(0)}(z, t)$. Then, from the state equation we have that $\vartheta^{(0)}=\vartheta^{(0)}(z, t)$. The form of the zero order thermodynamic variables depends on the (boundary conditions of the) particular problem under consideration. Assuming that they are constant (what is a limitation on the validity of the approximation) it. follows that:

$$
\begin{gathered}
\nabla \cdot u^{(0)}=0 \\
\rho^{(0)}\left(\mathbf{S} \frac{\partial u^{(0)}}{\partial t}+u^{(0)} \cdot \nabla u^{(0)}\right)+\nabla p^{(2)}=\frac{1}{\mathbf{R}} \nabla \cdot\left(2 \mu \varepsilon^{\prime}\left(u^{(0)}\right)\right)-\rho^{(1) \hat{z}} \\
\rho^{(0)} c_{p}^{(0)}\left(\frac{\partial \vartheta^{(1)}}{\partial t}+u^{(0)} \cdot \nabla \vartheta^{(1)}\right)=\frac{1}{P} \nabla \cdot\left(k \nabla \vartheta^{(1)}\right)+S Q
\end{gathered}
$$

\section{Finite element} approximation

839

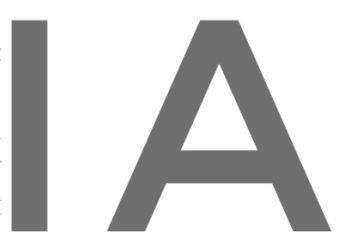


HFF

$18,7 / 8$

\section{0}

and the first order pressure is determined from equation (9) as $p^{(1)}=-\rho^{(0)} z$ to obtain:

$$
p^{(1)}=\rho^{(0)} \vartheta^{(1)}+\vartheta^{(0)} \rho^{(1)}
$$

$$
\rho^{(1)}=-\frac{\rho^{(0)}}{\vartheta^{(0)}} z-\frac{\rho^{(0)}}{\vartheta^{(0)}} \vartheta^{(1)}
$$

As it is usually done, a redefinition of the second order pressure permits to include the first term in the pressure gradient. Note that for ideal gases $\beta=1 / \vartheta$ and when the zero order thermodynamic fields are constant we have that $\rho^{(1)} \sim-\rho^{(0)} \beta^{(0)} \vartheta^{(1)}$.

In order to discuss some aspects of the numerical formulation considered, let us write down the incompressible Navier Stokes equations which are obtained in absence of heating and when the thermodynamic pressure is constant (i.e. when open flows or closed flows without addition of mass are considered):
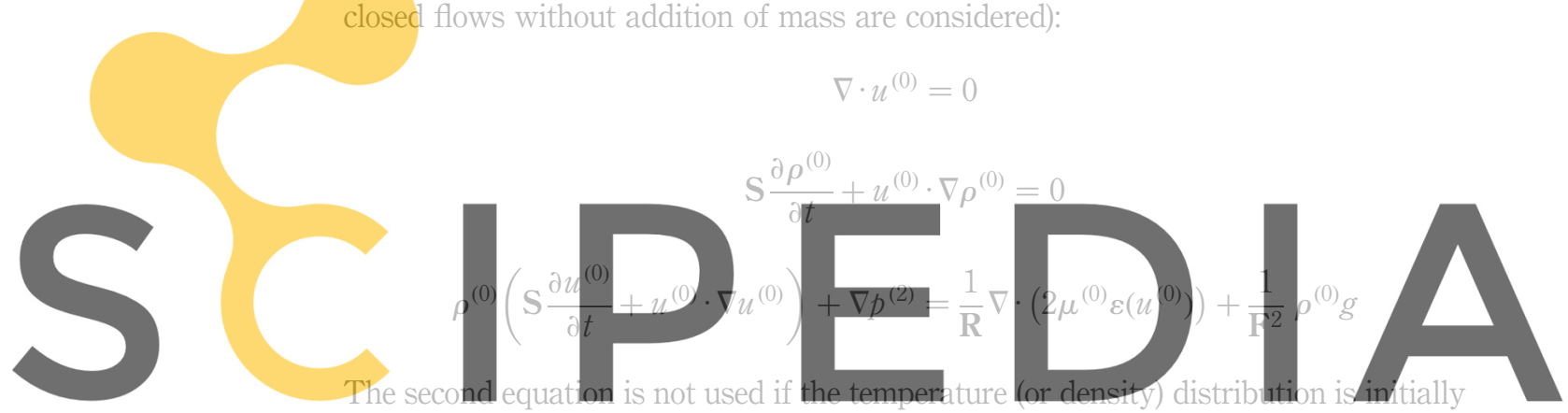

constant (it remains constant for all times).

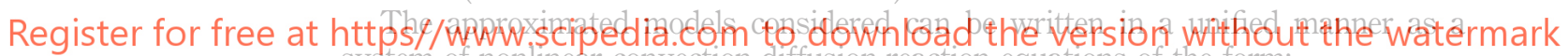
system of nonlinear convection-diffusion-reaction equations of the form:

$$
\mathbf{M} \frac{\partial U}{\partial t}+\mathscr{L}(U ; U)=\mathbf{F} \text { in } \Omega
$$

where:

$$
\mathscr{L}\left(U_{0} ; U\right):=\mathbf{A}_{i}\left(U_{0}\right) \frac{\partial U}{\partial x_{i}}-\frac{\partial}{\partial x_{i}}\left(\mathbf{K}_{i j} \frac{\partial U}{\partial x_{j}}\right)+\mathbf{S}\left(U_{0}\right) U
$$

and $U=(u, p, \vartheta), \mathbf{F}$ is a known vector of $n_{\mathrm{unk}}=n_{\mathrm{sd}}+2$ components and $\mathbf{M}, \mathbf{A}_{i}, \mathbf{K}_{i j}$, and $\mathbf{S}$ are flunk $n_{\text {unk }} \times n_{\text {unk }}$ matrices $\left(i, j=1, \ldots, n_{\mathrm{sd}}\right)$. The usual summation convention is implied in the last expression, with indices running from 1 to the number of space dimensions $n_{\text {sd. }}$. We shall refer to the terms of the left-hand-side (LHS) of this equation as the temporal, the convective, the diffusive and the reactive terms. Equation (13) needs to be supplied with appropriate boundary. and initial conditions.

The approximated models arc obtained considering the following expressions of the matrices and vectors involved in equation (13). They are written using dimensions, omitting the superscripts used for the asymptotic developments (the zero order thermodynamic pressure will be denoted by $p^{\text {th }}$ and $p$ will be used for the second order pressure) and for the $2 \mathrm{D}$ case $\left(n_{\mathrm{sd}}=2\right)$ as: 
- Incompressible Navier stokes equations:

$$
\begin{gathered}
\mathbf{M}=\left[\begin{array}{ccc}
\rho & 0 & 0 \\
0 & \rho & 0 \\
0 & 0 & 0
\end{array}\right], \quad \mathbf{A}_{i}(U)=\left[\begin{array}{ccc}
\rho u_{i} & 0 & \delta_{i 1} \\
0 & \rho u_{i} & \delta_{i 2} \\
\delta_{i 1} & \delta_{i 2} & 0
\end{array}\right] \\
\mathbf{K}_{i j}=\left[\begin{array}{ccc}
\mu \delta_{i j}+\mu \delta_{i 1} \delta_{j 1}+(2 \mu / 3) \delta_{i 1} \delta_{j 1} & \mu \delta_{i 2} \delta_{j 1}+(2 \mu / 3) \delta_{i 1} \delta_{j 2} & 0 \\
\mu \delta_{i 1} \delta_{j 2}+(2 \mu / 3) \delta_{i 2} \delta_{j 1} & \mu \delta_{i j}+\mu \delta_{i 2} \delta_{j 2}+(2 \mu / 3) \delta_{i 2} \delta_{j 2} & 0 \\
0 & 0 & 0
\end{array}\right]
\end{gathered}
$$$$
\mathrm{S}(U)=0, \quad \mathrm{~F}=0
$$

Finite element approximation

841

\section{- Boussinesq equations:}
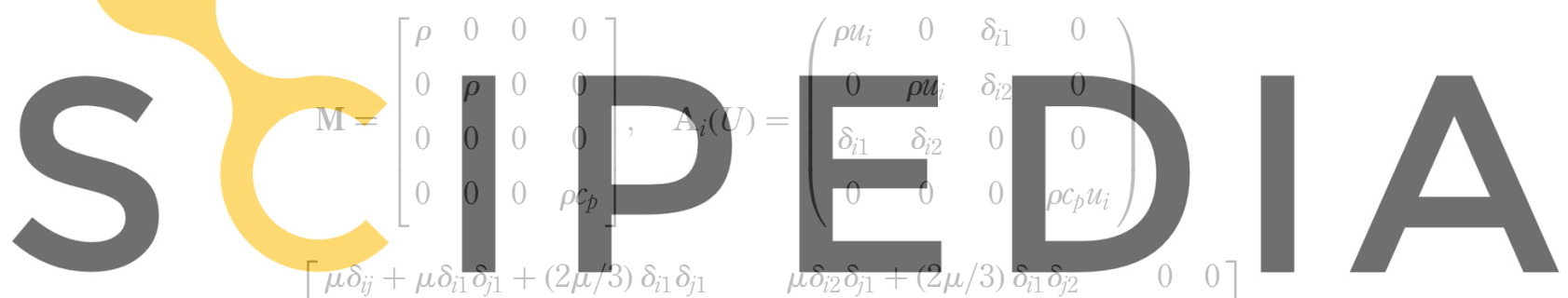

Register for free at https $/$ / w w w

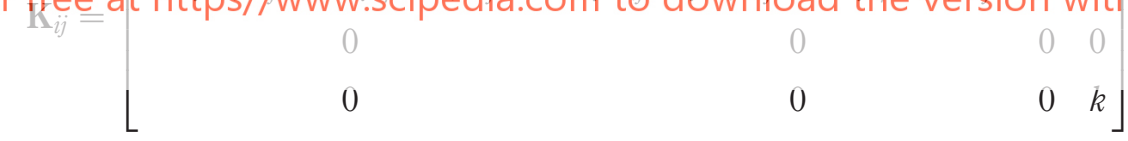

$$
\mathbf{S}(U)=\left[\begin{array}{cccc}
0 & 0 & 0 & \rho \beta g_{1} \\
0 & 0 & 0 & \rho \beta g_{2} \\
0 & 0 & 0 & 0 \\
0 & 0 & 0 & 0
\end{array}\right], \quad \mathbf{F}=\left[\begin{array}{l}
0 \\
0 \\
0 \\
Q
\end{array}\right]
$$

Note that in this case $\rho$ is not an unknown of the problem, but a constant physical property.

- Low Mach number model: in this case an ideal gas will be considered and the density is considered as a temperature dependent property what permits to write the continuity equation as:

$$
-\frac{\rho}{\vartheta} \frac{\partial \vartheta}{\partial t}+\frac{\rho}{p^{\text {th }}} \frac{\mathrm{d} \rho^{\text {th }}}{\mathrm{d} t}-\frac{\rho}{\vartheta} u \cdot \nabla \vartheta+\rho \nabla \cdot u=0
$$


HFF

$18,7 / 8$

842

This is used to write the matrices as:

$$
\mathbf{M}(U)=\left[\begin{array}{cccc}
\rho & 0 & 0 & 0 \\
0 & \rho & 0 & 0 \\
0 & 0 & 0 & -(1 / \vartheta) \\
0 & 0 & 0 & \rho c_{p}
\end{array}\right], \quad \mathbf{A}_{i}(U)=\left[\begin{array}{cccc}
\rho u_{i} & 0 & \delta_{i 1} & 0 \\
0 & \rho u_{i} & \delta_{i 2} & 0 \\
\delta_{i 1} & \delta_{i 2} & 0 & -(1 / \vartheta) u_{i} \\
0 & 0 & 0 & \rho c_{p} u_{i}
\end{array}\right]
$$$$
\mathbf{K}_{i j}=\left[\begin{array}{cccc}
\mu \delta_{i j}+\mu \delta_{i 1} \delta_{j 1}+(2 \mu / 3) \delta_{i 1} \delta_{j 1} & \mu \delta_{i 2} \delta_{j 1}+(2 \mu / 3) \delta_{i 1} \delta_{j 2} & 0 & 0 \\
\mu \delta_{i 1} \delta_{j 2}+(2 \mu / 3) \delta_{i 2} \delta_{j 1} & \mu \delta_{i j}+\mu \delta_{i 2} \delta_{j 2}+(2 \mu / 3) \delta_{i 2} \delta_{j 2} & 0 & 0 \\
0 & 0 & 0 & 0 \\
0 & 0 & 0 & k
\end{array}\right]
$$

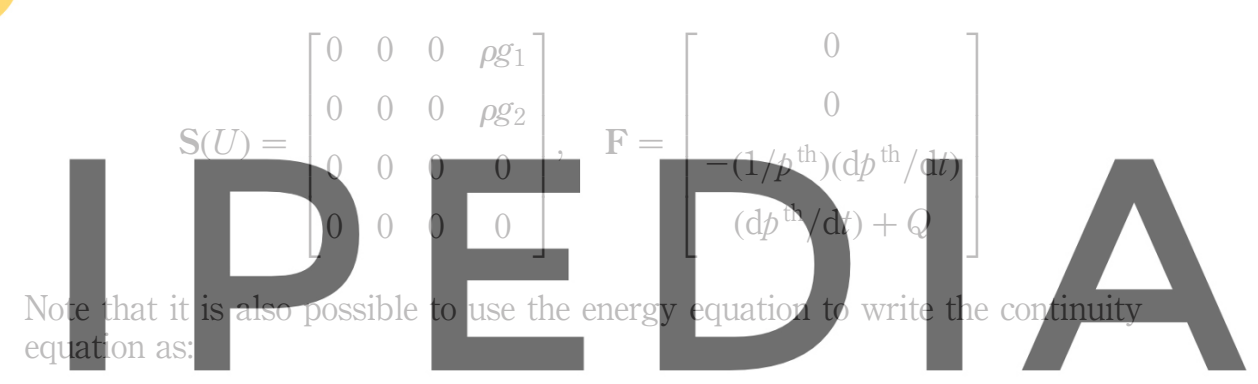

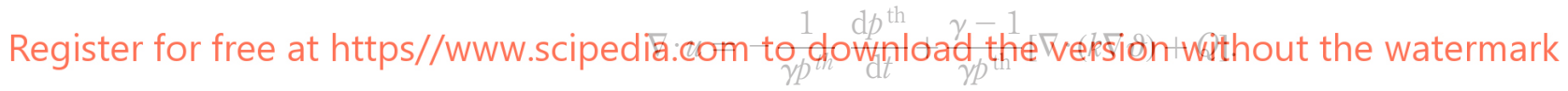

and that, as the density is temperature dependent, the temporal term is nonlinear.

Let us finally mention that boundary conditions are of the form:

$$
\begin{gathered}
u=u_{d} \text { on } \Gamma_{\mathrm{D}}^{u} \\
\vartheta=\vartheta_{d} \quad \text { on } \Gamma_{\mathrm{D}}^{\vartheta} \\
\sigma \cdot n=\left(-p I+2 \mu \varepsilon^{\prime}(u)\right) \cdot n=t \text { on } \Gamma_{N}^{u} \\
q \cdot n=-k n \cdot \nabla \theta=q_{n} \text { on } \Gamma_{N}^{\vartheta}
\end{gathered}
$$

where $\Gamma_{\mathrm{D}}^{\alpha}\left(\Gamma_{N}^{\alpha}\right)$ is the part of the domain boundary where Dirichlet (Neumann) boundary conditions are given and:

$$
\Gamma=\partial \Omega=\overline{\Gamma_{N}^{\alpha} \cup \Gamma_{\mathbf{D}}^{\alpha}}
$$

where $\alpha$ is either the velocity $u$ or the temperature $\vartheta$. Initial conditions have to be appended to close the problem. 


\section{Discrete approximation}

\subsection{Variational formulation}

Let us denote by $W$ the functional space where the solution is to be sought, by $W^{m, p}(\omega)$ the usual Sobolev spaces and, in particular $H^{m}(\omega):=W^{m, 2}(\omega)$ and by $L^{2}(\omega)$ the space of square integrable functions in a domain $\omega$. In the steady state case, the components of $u$ and $\vartheta$ must be $H^{1}(\Omega)$ functions satisfying the Dirichlet boundary conditions. The space of functions where these unknowns belong will be denoted by:

$$
V_{\mathbf{D}}^{u}(\Omega)=\left\{u \in\left[H^{1}(\Omega)\right]^{n_{\mathrm{sd}}}: u=u_{\mathbf{D}} \text { in } \Gamma_{\mathbf{D}}^{u}\right\}
$$

and:

$$
V_{\mathrm{D}}^{\vartheta}(\Omega)=\left\{\vartheta \in H^{1}(\Omega): \vartheta=\vartheta_{\mathrm{D}} \text { in } \Gamma_{\mathrm{D}}^{\vartheta}\right\}
$$

respectively, whereas $p$ must be an $L^{2}(\Omega)$ function $\left(L^{2}(\Omega) / \mathbb{R}\right.$ if $\left.\Gamma_{N}^{u}=\varnothing\right)$. When a transient problem defined in the interval $[0, T]$ is considered, the space of time dependent functions defined in a space $X$ whose norm is $L^{p}(0, T)$ will be denoted by $L^{p}(0, T ; X)$. Then the space $W$ is defined as:

$$
W=L^{2}\left(0, T, V_{\mathrm{D}}^{u}(\Omega)\right) \times L^{2}\left(0, T, V_{\mathrm{D}}^{\vartheta}(\Omega)\right) \times L^{1}\left(0, T, L^{2}(\Omega)\right)
$$

and $W_{0}$, the corresponding space of test functions, is defined as:
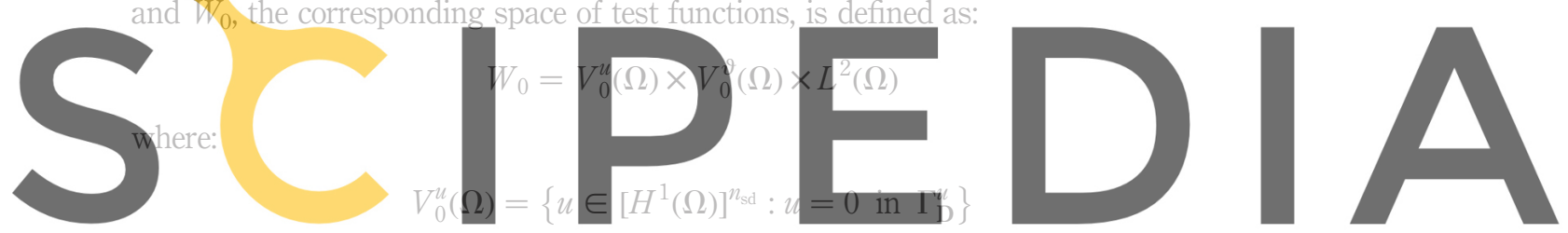

Register for free at https//www.scipedia.com to download the version without the watermark $V_{0}^{\vartheta}(\Omega)=\left\{\vartheta \in H^{1}(\Omega): \vartheta=0\right.$ in $\left.\Gamma_{\mathrm{D}}^{\vartheta}\right\}$

In order to write boundary conditions in a unified manner we split matrices $\mathbf{A}_{i}$ as $\mathbf{A}_{i}=\mathbf{A}_{i}^{\mathrm{c}}+\mathbf{A}_{i}^{f}$, where $\mathbf{A}_{i}^{\mathrm{c}}$ is the part of the convection matrices which is not integrated by parts and $\mathbf{A}_{i}^{f}$ the part that is integrated by parts. In the case of the Navier Stokes equations, this matrix contains pressure terms and is given by:

$$
\mathbf{A}_{i}^{f}=\left[\begin{array}{ccc}
0 & 0 & \delta_{i 1} \\
0 & 0 & \delta_{i 2} \\
0 & 0 & 0
\end{array}\right], \quad \mathbf{A}_{i}^{\mathrm{c}}=\mathbf{A}_{i}-\mathbf{A}_{i}^{f}
$$

Just to simplify the presentation, let us consider the simple case in which $\Gamma_{N}^{u}=\Gamma_{N}^{\vartheta}:=\Gamma_{N} \subset \partial \Omega$. In this case, boundary conditions can be written as:

$$
\mathbf{n}_{i} \mathbf{K}_{i j} \frac{\partial U}{\partial x_{j}}-\mathbf{n}_{i} \mathbf{A}_{i}^{f} U=\mathbf{T} \quad \text { in } \quad \Gamma_{N}
$$

and the weak form of the problem consists in finding $U \varepsilon W$ such that:

$$
B(U ; U, V)-L(V)=0 \quad \forall V \in W_{0}
$$




\section{$\mathrm{HFF}$}

$18,7 / 8$

\section{4}

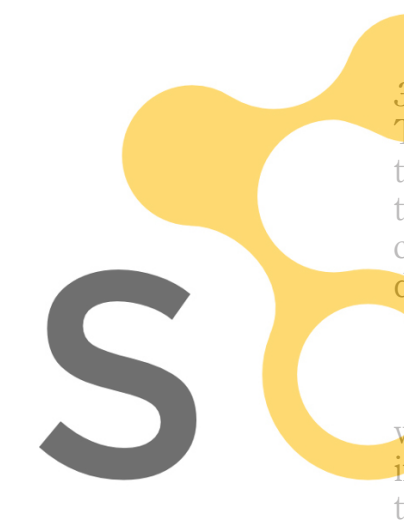

Register for free at htepsety wrowlemspedia.com to downloadthe version without the watermark

where the nonlinear form $B$ and the linear form $L$ are defined as:

$$
\begin{aligned}
B\left(U_{0} ; U, V\right):= & \left\langle V^{t}, \mathbf{M}\left(U_{0}\right) \frac{\partial U}{\partial t}+L\left(U_{0} ; U\right)\right\rangle \\
:= & \int_{\Omega} V^{t} \mathbf{M} \frac{\partial U}{\partial t}+\int_{\Omega} V^{t} \mathbf{A}_{i}^{c} \frac{\partial U}{\partial x_{i}}-\int_{\Omega} \frac{\partial}{\partial x_{i}}\left(V^{t} \mathbf{A}_{i}^{f}\right) U \\
& +\int_{\Omega} \frac{\partial V^{t}}{\partial x_{i}} \mathbf{K}_{i j} \frac{\partial U}{\partial x_{j}}+\int_{\Omega} V^{t} S U \\
& L(V):=\int_{\Omega} V^{t} \mathbf{F}+\int_{\Gamma_{N}} V^{t} T \mathrm{~d} \Gamma
\end{aligned}
$$

\subsection{Galerkin finite element approximation}

The Galerkin finite element approximation of this problem is standard. A partition of the domain $P_{h}=\{\mathbf{K}\}$ is a set of $n_{\mathrm{el}}$ elements $\mathbf{K}$ such that they cover the domain and they are cither disjoint or they share a complete face (edge). Based on this partition, and considering $\Omega$ be polyhedral (polygonal) the space $W$ is approximated by a finite

\section{dimensional space}

where $P$

if tetraht
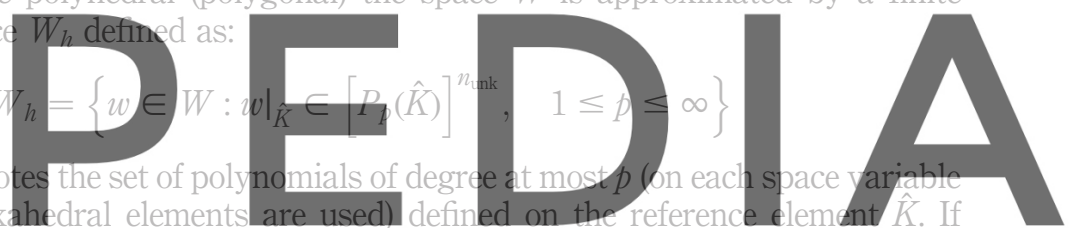

fetrahedral/hexahedral elements are used) defined one reference elendnth. If

$B\left(U_{h} ; U_{h}, V_{h}\right)-L\left(V_{h}\right)=0 \quad \forall V_{h} \in W_{0, h}$

It is well known that this formulation lacks stability when the diffusive terms are small, compared either to the convective or to the reactive terms. In simple scalar problems, this lack of stability can be measured in terms of dimensionless numbers. However, it is not possible to define such numbers for the general vector case. Likewise, since the quadratic form associated to $\mathbf{K}_{i j}$ is not positive definite, it is not possible to use equal interpolation for all the components of $U$. In our case, velocity-pressure pairs must satisfy the inf-sup condition. If the thermal coupling is strong, this could also be a source of numerical instabilities.

\subsection{Stabilized finite element approximation}

The purpose of this subsection is to describe the stabilized finite element formulation we employ to solve problem (14). This formulation is based on the subgrid scale method with an algebraic approximation to the subscales (Hughes, 1995). This method, originally devised for linear steady problems, has been extended to the transient nonlinear case in Codina (2002), Codina et al. (2007) and Codina and Principe (2007), where a nonlinear time dependent stabilization was developed. A linear steady stabilization technique can be obtained starting from the following linearized version of the problem: 


$$
B\left(U_{0, h} ; U_{h}, V_{h}\right)-L\left(V_{h}\right)=0 \quad \forall V_{h} \in W_{0, h}
$$

where $U_{0, h}$ is a known finite element function.

3.3.1 The subgrid scale approach. Let us split the continuous space $W$ as $W=W_{h} \oplus \tilde{W}$, where $\tilde{W}_{\text {can }}$ be in principle any space to complete $\mathrm{W}_{h}$ in W. To fix ideas, we may think of $\tilde{W}$ as the orthogonal complement of $W_{h}$, with respect to the $L^{2}$ inner product in $W$. Since $\tilde{W}$ represents the component of $W$ which is not reproduced by the finite element space, we call it the space of subscales or subgrid scales. The continuous equation (14) can now be written as the system:

$$
\begin{gathered}
B\left(U_{o, h} ; U_{h}, V_{h}\right)+B\left(U_{0, h} ; \tilde{U}, V_{h}\right)=L\left(V_{h}\right) \quad \forall V_{h} \in W_{h} \\
B\left(U_{o, h} ; U_{h}, \tilde{V}\right)+B\left(U_{0, h} ; \tilde{U}, \tilde{V}\right)=L(\tilde{V}) \quad \forall \tilde{V} \in \tilde{W}
\end{gathered}
$$

where $U=U_{h}+\tilde{U}^{*}$ and $U_{h} \in \mathbf{W}_{h}, \tilde{U} \in \tilde{W}$. The nonlinear stabilization naturally appears if the splitting is also considered in $U_{0}$ by taking $U_{0}=U_{0, h}+\tilde{U}_{0}$.

It is useful for the following to introduce the notation:

$$
\int_{\Omega^{\prime}}:=\sum_{\mathbf{K} \in P_{h}} \int_{\mathbf{K}}, \quad \int_{\Gamma^{\prime}}:=\sum_{\mathbf{K} \in P_{h}} \int_{\partial \mathbf{K}}
$$

Let us assume that the solution of the continuous problem $U$ is smooth. Integrating by parts within each element domain it is found that problems (17) and (18) can be written as:

$$
\begin{gathered}
B\left(U_{0, h} ; U_{h}, V_{h}\right)+\int_{\Gamma^{\prime}}\left(\mathbf{K}_{i j} \frac{\partial V_{h}^{t}}{\partial x_{i}}-\mathbf{A}_{i}^{f} V_{h}^{t}\right) \tilde{U}_{i} \mathrm{~d} \Gamma \\
+\int_{\Omega} V^{t} \mathbf{M}\left(U_{o, h}\right) \frac{\partial \tilde{U}}{\partial t}+\int_{\Omega^{\prime}}\left[\mathscr{L}^{*}\left(U_{0, h} ; V_{h}\right)\right]^{t} \tilde{U}=\mathscr{L}\left(V_{h}\right), \\
\int_{\Gamma^{\prime}} \tilde{V}^{t} n_{i}\left(\mathbf{K}_{i j} \frac{\partial}{\partial x_{i}}\left(U_{h}+\tilde{U}\right)-\mathbf{A}_{i}^{f}\left(U_{h}+\tilde{U}\right)\right) \mathrm{d} \Gamma+\int_{\Omega} V^{t} \mathbf{M}\left(U_{0, h}\right) \frac{\partial \tilde{U}}{\partial t} \\
+\int_{\Omega^{\prime}} \tilde{V}^{t} \mathscr{L}\left(U_{0, h} ; \tilde{U}\right)=\int_{\Omega^{\prime}} \tilde{V}^{t}\left[\mathbf{F}-\mathbf{M}\left(U_{0, h}\right) \frac{\partial U_{h}}{\partial t}-\mathscr{L}\left(U_{0, h} ; U_{h}\right)\right]
\end{gathered}
$$

where $\mathscr{L}^{*}$ is the adjoint operator of $\mathscr{L}$ with homogeneous Dirichlet conditions. Note that when the splitting of $U_{0}$ is considered, the adjoint operator $\mathscr{L}^{*}$ depends not only on $U_{0, h}$, but also on $\tilde{U}_{0}$, and this is the reason why we refer to a nonlinear stabilization technique.

Equation (20) is equivalent to finding $\tilde{U} \in \tilde{W}$ such that:

$$
\begin{gathered}
\mathbf{M}\left(U_{0, h}\right) \frac{\partial \tilde{U}}{\partial t}+\mathscr{L}\left(U_{0, h} ; \tilde{U}\right)=\mathbf{F}-\mathbf{M}\left(U_{0, h}\right) \frac{\partial U_{h}}{\partial t}-\mathscr{L}\left(U_{0, h} ; U_{h}\right)+V_{h, \text { ort }} \text { in } \mathbf{K} \\
\tilde{U}=\tilde{U}_{\text {ske }} \text { in } \partial \mathbf{K}
\end{gathered}
$$

for any $\mathbf{K} \in \Pi_{h}$, where $V_{h \text {,ort }}$ is obtained from the condition that $\tilde{U}$ must belong to $\tilde{W}$ (and not to the whole space $W$ ) and $\tilde{U}_{\text {ske }}$ is a function defined on the element boundaries 
$\mathrm{HFF}$

$18,7 / 8$

846

and such that:

$$
\mathbf{q}_{n}:=\mathbf{n}_{i}\left(\mathbf{K}_{i j} \frac{\partial}{\partial x_{i}}\left(U_{h}+\tilde{U}\right)-\mathbf{A}_{i}^{f}\left(U_{h}+\tilde{U}\right)\right)
$$

is continuous across interelement boundaries, that is to say, the normal component of the fluxes of $U$ is continuous across these boundaries. Observe that due to this fact the first term in the LHS of vanishes. We call $\tilde{U}_{\text {ske }}$ the skeleton of $\tilde{U}$.

Problems (17) and (18) are exactly equivalent to equations (19), (21) and (22). The approximate problem is defined by the way in which problems (21) and (22) are solved as well as by the way in which the functions $V_{h \text {,ort }}$ and $\tilde{U}_{\text {ske }}$ are taken. We restrict ourselves to a particularly simple case although several other possibilities have been already considered. In the previous work (Codina, 2000b), the subscales are taken orthogonal to the finite element space, in Codina (2002) and Codina et al. (2007), they are allowed to depend on time and in Codina et al. (2007) and Codina and Principe (2007) the subscale splitting of $U_{0}$ has been considered, giving rise to a nonlinear stabilization technique.

3.3.2 Algebraic approximation to the subscales. The simplest way to approximate problems (21) and (22) are to take:

$$
\tilde{U} \approx \boldsymbol{\tau}\left[\mathbf{F}-\mathbf{M}\left(U_{0, h}\right) \frac{\partial U_{h}}{\partial t}-\mathscr{L}\left(U_{0, h} ; U_{h}\right)\right]
$$

as the solution of this problem, where $\boldsymbol{\tau}$ is a $n_{\mathrm{unk}} \times n_{\mathrm{unk}}$ matrix defined within each element domain that has to be determined. We shall refer to it as the matrix of stabilization parameters.

The approximation given by equation (24) has an implicit assumption on the function $\tilde{U}_{\text {ske }}$ and the space $\tilde{W}$, and therefore on the function $V_{h \text {,ort }}$. It also assumes that:

$$
\frac{\partial \tilde{U}}{\partial t}=0
$$

what in Codina (2002) and Codina et al. (2007) is referred to as quasistatic subscales. In general, $\tilde{U}$ will be discontinuous across interelement boundaries, so that the fluxes given by equation (23) will not even be well defined. However, from equation (19) it is observed that, except for the boundary integral, only the component of $\tilde{U}$ in $L\left(U_{0, h} ; W_{h}\right)$ is needed, where $L\left(U_{0, h} ; W_{h}\right)$ is the space of functions of the form $L\left(U_{0, h} ; V_{h}\right)$, with $V_{h} \in W_{h}$. We may think of equation (24) as the approximation to this component.

To close the approximation, we neglect the interelement boundary terms in equation (19), so that the problem that has to be solved is finally:

$$
B\left(U_{0, h} ; U_{h}, V_{h}\right)+\int_{\Omega^{\prime}}\left[\mathscr{L}^{*}\left(U_{0, h} ; V_{h}\right)\right]^{t} \tilde{U}=\mathscr{L}\left(V_{h}\right)
$$

with $\tilde{U}$ given by equation (24). With all these assumptions, we have arrived to a method proposed previously (Hughes, 1995) using different arguments. In particular, equation (24) was derived from an approximation to the Green's function of the problem. This method was also considered in the literature (Franca et al., 1992) and derived for the scalar diffusion-reaction equation by using bubble functions (Franca and Farhat, 1995). 
The way to compute matrix $\boldsymbol{\tau}$ is general situations is still not clear. Traditionally, the way to proceed has been to obtain particular expressions for simplified problems and then to extend them to more complex situations. Very often, the appropriateness of the expressions thus obtained have been confirmed by convergence analysis.

A simple approximation for $\boldsymbol{\tau}$ (Codina, 2002) (in the 2D case) is given by:

$$
\boldsymbol{\tau}=\operatorname{diag}\left(\boldsymbol{\tau}_{1}, \boldsymbol{\tau}_{1}, \boldsymbol{\tau}_{2}, \boldsymbol{\tau}_{3}\right)
$$

where:

$$
\boldsymbol{\tau}_{1}=\left[c_{1} \frac{\mu}{h^{2}}+c_{2} \frac{\rho\left|u_{0, h}\right|}{h}\right]^{-1}, \quad \boldsymbol{\tau}_{2}=\frac{h^{2}}{c_{1} \boldsymbol{\tau}_{1}}, \quad \boldsymbol{\tau}_{3}=\left[c_{1} \frac{k}{h^{2}}+c_{2} \frac{\rho\left|u_{0, h}\right|}{h}\right]
$$

where $c_{1}$ and $c_{2}$ are algorithmic constants that we take $c_{1}=4$ and $c_{2}=2$ for linear elements and $u_{0, h}$ is the velocity component of $U_{0, h}$.

\subsection{Time discretization}

All problems presented above have a common structure: an equation for the velocity field coupled with a constraint that determines the pressure and (possibly) a coupled transport equation for the temperature. Owing to this implicit nature of the pressure field, we consider implicit time integration schemes. Given the solution at time $t^{n}=n \delta t$, denoted as $U^{n}$ and $\alpha$ a parameter whose value can be 1 (first order scheme) or $1 / 2$ (second order scheme), the solution at time $t^{n+1}$ is found as the solution of:

$$
\begin{aligned}
\int_{\Omega} & V_{h}^{t} M \delta_{t} U_{h}^{n}+\int_{\Omega} V_{h}^{t} \mathbf{A}_{i}^{c} \frac{\partial U_{h}^{n+\alpha}}{\partial x_{i}}-\int_{\Omega} \frac{\partial}{\partial x_{i}}\left(V_{h}^{t} \mathbf{A}_{i}^{f}\right) U_{h}^{n+\alpha}+\int_{\Omega} \frac{\partial V_{h}^{t}}{\partial x_{i}} \mathbf{K}_{i j} \frac{\partial U_{h}^{n+\alpha}}{\partial x_{j}} \\
& +\int_{\Omega} V_{h}^{t} \mathbf{S} U_{h}^{n+\alpha}-\int_{\Omega^{\prime}}\left[\mathscr{L}^{*}\left(U_{0, h} ; V_{h}\right)\right]^{t} \tau\left[\mathbf{M}\left(U_{0, h}\right) \delta_{t} U_{h}^{n}+\mathscr{L}\left(U_{0, h} ; U_{h}^{n+\alpha}\right)\right] \\
= & \int_{\Omega} V^{t} \mathbf{F}^{n+\alpha}+\int_{\Gamma_{N}} V^{t} T^{n+\alpha} \mathrm{d} \Gamma+\int_{\Omega^{\prime}}\left[\mathscr{L}^{*}\left(U_{0, h} ; V_{h}\right)\right]^{t} \tau F^{n+\alpha}
\end{aligned}
$$

where:

$$
U^{n+\alpha}=\alpha U^{n+1}-(1-\alpha) U^{n}, \quad \delta_{t} U_{h}^{n}=\frac{\delta U_{h}^{n}}{\delta t} \text { and } \delta U_{h}^{n}=U^{n+1}-U^{n} .
$$

The final discrete problem to be solved is obtained when $U_{0, h}=U_{h}^{n+\alpha}$ and the algorithm to solve it is described in the following section.

\section{Numerical implementation}

\subsection{Linearization and line search strategy}

The discrete approximation described in the previous section leads to a nonlinear system of algebraic equations for the nodal values of $U_{h}^{n+\alpha}$, which are denoted by the same character (but without the subscript $h$ ). This nonlinear problem can be written as:

$$
[L+N(U)] U=\mathbf{R}
$$

where $L$ is the linear part of the operator and $N$ the nonlinear one and $\mathbf{R}$ the force vector. Therefore, we look for the roots of the function:

$$
H(U)=[L+N(U)] U-\mathbf{R}
$$


$\mathrm{HFF}$

$18,7 / 8$

and we consider fixed points linearizations of the form:

$$
U^{k}=G\left(U^{k-1}\right)
$$

where:

$$
G(U)=\mathbf{D}^{-1}(\mathbf{D} U-H(U))
$$

for some matrix $\mathbf{D}$ to be defined in the following and which may depend on the iteration step. Then, using a superscript for the iteration counter the iterative scheme reads:

$$
\mathbf{D}\left(U^{i+1}-U^{i}\right)+H\left(U^{i}\right)=0
$$

or:

$$
\mathbf{D}\left(U^{i+1}-U^{i}\right)+[L+N(U)] U^{i}-\mathbf{R}=0
$$

Different choices of $\mathbf{D}$ led to different schemes:

- The classical Picard scheme is obtained by taking:

$$
\mathbf{D}=[L+N(U)]
$$

from where the problem to be solved is:

$$
\left[L+N\left(U^{i}\right)\right] U^{i+1}=\mathbf{R}
$$

- The Newton scheme is obtained taking:

$$
\mathbf{D}=H^{\prime}(U)
$$

where $H^{\prime}$ is the Jacobian of $H$, from where:

$$
H^{\prime}\left(U^{i}\right)\left(U^{i+1}-U^{i}\right)+H\left(U^{i}\right)=0
$$

Sometimes, a modified Newton scheme is obtained by taking:

$$
\mathbf{D}=H^{\prime}\left(U^{0}\right)
$$

- In the case of a steady state problem (just to fix ideas), another option is to take:

$$
\mathbf{D}=\frac{1}{\varepsilon} \mathbf{M}
$$

to obtain:

$$
\frac{1}{\varepsilon} \mathbf{M}\left(U^{i+1}-U^{i}\right)+\left[L+N\left(U^{i}\right)\right] U^{i}=\mathbf{R}
$$

This scheme produces the same iterates that an explicit temporal integration of the equations, what shows how a temporal evolution can be considered as a fixed point scheme for the solution of a nonlinear problem. 
- In a similar way, if we take:

$$
\mathbf{D}=\frac{1}{\varepsilon} \mathbf{M}+\left[L+N\left(U^{i}\right)\right]
$$

we obtain a semi-implicit temporal evolution:

$$
\frac{1}{\varepsilon} \mathbf{M}\left(U^{i+1}-U^{i}\right)+\left[L+N\left(U^{i}\right)\right] U^{i+1}=\mathbf{R}
$$

The convergence rate of the method depend on how contractive the mapping $G$ is. Precisely (Kolmogorov and Fomin, 1999), if there exists $a$ such that:

$$
|G(U)-G(V)| \leq \alpha|U-V|
$$

the mapping $G$ has only one fixed point $U^{*}$ and the iterative scheme:

$$
U^{i+1}=G\left(U^{i}\right)
$$

converges at a rate given by the estimator:

$$
\left|U^{i}-U_{*}\right| \leq \frac{\alpha^{i}}{1-\alpha}\left|U^{0}-U^{1}\right|
$$

In particular, if the Jacobian of $G$ is bounded we can take:

$$
\alpha=\left|G^{\prime}(U)\right|
$$

and using equation (25), we have (for a fixed $\mathbf{D}$ ):

$$
G^{\prime}(U)=\mathbf{I}-\mathbf{D}^{-1} H^{\prime}(U)
$$

The Newton method is based on a choice that makes $G$ highly contractive but only in some neighborhood of the solution, which is the reason why it requires a good initial condition. If a temporal evolution is used to solve the problem, we have that $\mathbf{D}^{-1}=\varepsilon M^{-1}$, which shows that, when $\varepsilon \rightarrow 0,\left|G^{\prime}(U)\right| \rightarrow 1$ making the iterative procedure very slow. Note that if:

$$
\mathbf{D}=\frac{1}{\varepsilon} \mathbf{M}+H^{\prime}(U)
$$

when $\varepsilon \rightarrow 0$, we have that $\mathbf{D}^{-1} \rightarrow \varepsilon M^{-1}$ and if $\varepsilon \rightarrow \infty$, we have $\mathbf{D}^{-1} \rightarrow\left[H^{\prime}(U)\right]^{-1}$ as in the Newton method.

The problem of the sensitivity of the Newton method with the initial condition can be partially solved using globally convergent methods (methods that converge for almost any initial guess) which can be developed by adding a line search strategy (Press et al., 1992; Dennis and Schnabel, 1983; Kelley, 1999). As a root of $H$ is a minimum of the function:

$$
f(U)=\frac{1}{2} H(U) \cdot H(U)
$$


$\mathrm{HFF}$

$18,7 / 8$

\section{0}

one may be tempted to apply a minimization algorithm to find the solution, but this is not a good idea because there could be a local minimum of $f$ that is not a root of $H$. However, this function is used to find the optimal parameter of advance. The direction of advance $P$ is found solving the linear system:

$$
D P=-H\left(U^{i}\right)
$$

and the next iterate is taken as:

$$
U^{i+1}=U^{i}+s P
$$

where $s$ is the advancing parameter whose calculation is as follows. The step is accepted if the function $f$ decreases at least a small fraction of the decrease given by a linear approximation at $s=0$. This condition, known as Armijo rule, can be written as:

$$
f\left(U^{k+1}\right) \leq f\left(U^{k}\right)+\xi \nabla f \cdot\left(U^{k+1}-U^{k}\right)
$$

where $\xi$ is a parameter of the method taken to be $10^{-4}$, and prevents the algorithm to find a local minimum of $f$. This criterion is applied when a Newton type linearization of the problem is used because in this case $\mathbf{D}=H^{\prime}(U)$ and then:

$$
\nabla f \cdot P=\left[H(U) \cdot H^{\prime}(U)\right] \cdot P=\left[H(U) \cdot H^{\prime}(U)\right] \cdot\left[-\mathbf{D}^{-1} H(U)\right]=-H(U) \cdot H(U)
$$

In this case, one first tries $s=1$ since if we are close to the solution using a Newton type linearization we will have a high rate of convergence (quadratic if the exact Jacobian is used). If the step is not accepted, a new value of $s$ is tested. This value is found using a cubic model based on the values of $f\left(U_{h}^{k}+s P\right)$ previously computed (Press et al., 1992; Dennis and Schnabel, 1983; Kelley, 1999) but it can be simply taken as a fraction of the previous one. This method can select a step that is too small (this happens when a local minimum of $f$ has been found). In such a case, the method has to be restarted. We do so performing a Picard step, i.e. changing the searching direction.

When a Picard type scheme is used, we accept the step when:

$$
f\left(U^{k+1}\right) \leq f\left(U^{k}\right)
$$

Again, we first try $s=1$ and if the step is not accepted some smaller values of $s$ are tested and the one that gives the minimum value of $f$ is kept.

\subsection{Linearized flow equations}

In the previous development, matrix $\mathbf{D}$ is taken as an approximation to the exact derivative of the function $H$. When we apply this to the flow equations we consider, we always evaluate the stabilization parameters as well as the adjoint operator using the previous iterate, but a full linearization of the operator $L$ can be considered. This linearization can be written in-terms of the linearized advection and reaction matrices, $\mathbf{A}_{i}^{\operatorname{lin}}\left(U_{h}^{i-1}\right)$ and $\mathbf{S}^{\operatorname{lin}}\left(U_{h}^{i-1}\right)$, as well as of the resulting forcing vector $\mathbf{F}^{\operatorname{lin}}\left(U_{h}^{i-1}\right)$. The expression of these matrices and vector is given below for different flow cases. Here, we have explicitly displayed their dependency with respect to the known iterate $U_{h}^{i-1}$ of $U_{h}$.

Having introduced these terms, the linearized differential operator applied to the finite element unknown is: 


$$
\mathscr{L}^{\operatorname{lin}}\left(U_{h}^{i-1} ; U_{h}^{i}\right):=\mathbf{A}_{i}^{\operatorname{lin}}\left(U_{h}^{i-1}\right) \frac{\partial U_{h}^{i}}{\partial x_{i}}-\frac{\partial}{\partial x_{i}}\left(\mathbf{K}_{i j} \frac{\partial U_{h}^{i}}{\partial x_{j}}\right)+\mathbf{S}^{\operatorname{lin}}\left(U_{h}^{i-1}\right) U_{h}^{i}
$$

The fully discrete stabilized problem now reads:

$$
\begin{aligned}
& \int_{\Omega} V_{h}^{t} \mathbf{M} \delta_{t} U_{h}^{n, i}+\int_{\Omega} V_{h}^{t} \mathbf{A}_{i}^{\operatorname{lin}, c} \frac{\partial U_{h}^{n+\alpha, i}}{\partial x_{i}}-\int_{\Omega} \frac{\partial}{\partial x_{i}}\left(V_{h}^{t} \mathbf{A}_{i}^{\operatorname{lin}, f}\right) U_{h}^{n+\alpha, i} \\
& +\int_{\Omega} \frac{\partial V_{h}^{t}}{\partial x_{i}} \mathbf{K}_{i j} \frac{\partial U_{h}^{n+\alpha, i}}{\partial x_{i}}+\int_{\Omega} V_{h}^{t} \mathbf{S}^{\operatorname{lin}} U_{h}^{n+\alpha, i} \\
& \quad+\int_{\Omega^{\prime}}\left[\mathscr{L}^{*}\left(U_{h}^{n+\alpha, i-1} ; V_{h}\right)\right]^{t} \boldsymbol{\tau}\left[\mathbf{M} \delta_{t} U_{h}^{n, i}+\mathscr{L}^{\operatorname{lin}}\left(U_{h}^{n+\alpha, i-1} ; U_{h}^{n+\alpha, i}\right)\right] \\
& \quad+\int_{\Omega} V^{t} \mathbf{F}^{\operatorname{lin}}+\int_{\Gamma_{N}} V^{t} T^{n+\alpha} \mathrm{d} \Gamma+\int_{\Omega^{\prime}}\left[\mathscr{L}^{*}\left(U_{h}^{n+\alpha, i-1} ; V_{h}\right)\right]^{t} \boldsymbol{\tau} \mathbf{F}^{\operatorname{lin}}
\end{aligned}
$$

where it is understood that the stabilization parameters in matrix $\boldsymbol{\tau}$ and matrices $\mathbf{M}$, $\mathbf{A}_{i}^{\operatorname{lin}}, \mathbf{S}^{\text {lin }}$ and $\mathbf{F}^{\text {lin }}$ are calculated using $U_{h}^{n+\alpha, i-1}$.

It remains to give the expression for $\mathbf{A}_{i}^{\operatorname{lin}}, \mathbf{S}^{\text {lin }}$ and $\mathbf{F}^{\text {lin }}$. To this end, let us define a set of parameters $\lambda_{i j}$ that can take the value 0 or 1 . For $i=1$, we will use them to write the linearized momentum equation, for $i=2$ the continuity equation and for $i=3$ the energy equation. The linearized matrices are given for each flow model as follows:

- Navier Stokes equations:

$$
\begin{gathered}
\mathbf{A}_{i}^{\operatorname{lin}}(U)=\left[\begin{array}{ccc}
\rho u_{i} & 0 & \delta_{i 1} \\
0 & \rho u_{i} & \delta_{i 2} \\
\delta_{i 1} & \delta_{i 2} & 0
\end{array}\right], \\
\mathbf{S}^{\operatorname{lin}}(U)=\left[\begin{array}{ccc}
\lambda_{11} \rho \partial_{1} u_{1} & \lambda_{11} \rho \partial_{2} u_{1} & 0 \\
\lambda_{11} \rho \partial_{1} u_{2} & \lambda_{11} \rho \partial_{2} u_{2} & 0 \\
0 & 0 & 0
\end{array}\right], \\
\mathbf{F}^{\operatorname{lin}}(U)=\left[\begin{array}{cc}
\lambda_{11} \rho u \cdot \nabla u_{1}+g_{1} \\
\lambda_{11} \rho u \cdot \nabla u_{2}+g_{2} \\
0
\end{array}\right] .
\end{gathered}
$$

- Boussinesq equations:

$$
\mathbf{A}_{i}^{\operatorname{lin}}(U)=\left[\begin{array}{cccc}
\rho u_{i} & 0 & \delta_{i 1} & 0 \\
0 & \rho u_{i} & \delta_{i 2} & 0 \\
\delta_{i 1} & \delta_{i 2} & 0 & 0 \\
0 & 0 & 0 & \rho u_{i}
\end{array}\right]
$$




\section{$\mathrm{HFF}$}

$18,7 / 8$

852

$$
\mathbf{S}^{\operatorname{lin}}(U)=\left[\begin{array}{cccc}
\lambda_{11} \rho \partial_{1} u_{1} & \lambda_{11} \rho \partial_{2} u_{1} & 0 & \lambda_{13} \rho \beta g_{1} \\
\lambda_{11} \rho \partial_{1} u_{2} & \lambda_{11} \rho \partial_{2} u_{2} & 0 & \lambda_{13} \rho \beta g_{2} \\
0 & 0 & 0 & 0 \\
\lambda_{31} \rho \partial_{1} \vartheta & \lambda_{31} \rho \partial_{2} \vartheta & 0 & 0
\end{array}\right]
$$

$$
\mathbf{F}^{\operatorname{lin}}(U)=\left[\begin{array}{c}
\lambda_{11} \rho u \cdot \nabla u_{1}-\left(1-\lambda_{13}\right) \rho \beta g_{1} \vartheta+\rho \beta \vartheta_{0} g_{1} \\
\lambda_{11} \rho u \cdot \nabla u_{2}-\left(1-\lambda_{13}\right) \rho \beta g_{1} \vartheta+\rho \beta \vartheta_{0} g_{2} \\
0 \\
\lambda_{31} \rho u \cdot \nabla \vartheta+Q
\end{array}\right] \text {. }
$$

- Low Mach number equations:

$$
\begin{aligned}
& \mathbf{A}_{i}^{\operatorname{lin}}(U)=\left[\begin{array}{cccc}
\rho u_{i} & 0 & \delta_{i 1} & 0 \\
0 & \rho u_{i} & \delta_{i 2} & 0 \\
\delta_{i 1} & \delta_{i 2} & 0 & -\frac{\lambda_{21}}{\vartheta} u_{i} \\
0 & 0 & 0 & \rho u_{i}
\end{array}\right], \\
& \mathbf{S}^{\operatorname{lin}}(U)=\left[\begin{array}{cccc}
\lambda_{11} \rho \partial_{1} u_{1} & \lambda_{11} \rho \partial_{2} u_{1} & 0 & \frac{\rho}{\vartheta}\left(-\lambda_{12} u \cdot \nabla u_{1}+\lambda_{13} g_{1}\right) \\
\lambda_{11} \rho \partial_{1} u_{2} & \lambda_{11} \rho \partial_{2} u_{2} & 0 & \frac{\rho}{\vartheta}\left(-\lambda_{12} u \cdot \nabla u_{2}+\lambda_{13} g_{2}\right) \\
-\frac{\lambda_{22}}{\vartheta} \partial_{1} \vartheta & -\frac{\lambda_{22}}{\vartheta} \partial_{2} \vartheta & 0 & \frac{\lambda_{23}}{\vartheta} u \cdot \nabla \vartheta \\
\lambda_{31} \rho \partial_{1} \vartheta & \lambda_{31} \rho \partial_{2} \vartheta & 0 & -\frac{\rho}{\theta} \lambda_{32} u \cdot \nabla \vartheta
\end{array}\right], \\
& \mathbf{F}^{\operatorname{lin}}(U)=\left[\begin{array}{c}
\left(\lambda_{11}-\lambda_{12}\right) \rho u \cdot \nabla u_{1}+\left(1+\lambda_{13}\right) \rho g_{1} \\
\left(\lambda_{11}-\lambda_{12}\right) \rho u \cdot \nabla u_{2}+\left(1+\lambda_{13}\right) \rho g_{2} \\
\left(1-\lambda_{21}-\lambda_{22}+\lambda_{23}\right)(1 / \vartheta) u \cdot \nabla \vartheta \\
\left(\lambda_{31}-\lambda_{22}\right) \rho u \cdot \nabla \vartheta+Q
\end{array}\right] .
\end{aligned}
$$

The parameters $\lambda_{11}$ and $\lambda_{12}$ correspond to the linearization of the convective term in the momentum equation $\left(\lambda_{11}=\lambda_{12}=1\right.$ would be Newton's method, whereas other options would be fixed point methods), whereas $\lambda_{13}$ is used to decide whether the buoyancy term is treated in a coupled or in a block iterative way. Likewise, $\lambda_{2 j}, j=1,2,3$, determine both the linearization of the term $(1 / \vartheta) u \cdot \nabla \vartheta\left(\lambda_{2 j}=1\right.$ would be full Newton's method) and the possibility to treat this term in a staggered way $\left(\lambda_{2 j}=0\right)$. Finally, $\lambda_{3 j}, j=1,2$, play the same role for the energy equation as $\lambda_{1 j}, j=1,2$, for the momentum equation. 


\section{Numerical examples}

In this section, we present three examples. This first one is the natural convection in a 2D closed cavity and, as it is a well-known benchmark for thermally coupled flows, we use it to test different numerical strategies proposed here. The second one is a $2 \mathrm{D}$ time dependent heated channel presented in Martinez and Gartling (2004) as a simplified version of what occurs in a chemical vapor deposition (CVD) reactor. CVD flow problems, reviewed in Jensen et al. (1991), present much of the physics of the Poiseuille-Rayleigh-Benard flow problem reviewed in Nicolas (2002), that consists of a channel with a prescribed Poiseuille velocity profile on the inlet and prescribed temperatures on the upper and lower walls. This example is included to illustrate the models considered as well as to point out the importance of outflow boundary conditions. The final example is the application of the developed approximation to a fire in a tunnel considering the fire as a fixed source of energy. The detailed combustion mechanism is not taken into account.

\subsection{Natural convection in a cavity}

The natural convection in a cavity is a standard benchmark for numerical methods on thermally coupled flows. It was initially devised for Boussinesq flows (Davis and Jones, 1983) and later for low Mach number flows (Le Quéré and Paillére, 2000). The problem is shown in Figure 1.

First of all, let us mention the conditions for the validity of the approximations in this example. As this is a natural convection problem, a velocity scale must be chosen. Taking, for example, the viscous scale and using the benchmark specifications (Le Quéré and Paillére, 2000) gives a Mach number of $2.2 \times 10^{-5}$, allowing the use of the zero Mach number equations. The conditions of applicability of the Boussinesq approximation need some care. In this case, the zero order temperature and density must be constants. In order to have this reference state, the (dimensionless) temperature difference between vertical walls must vanish. Finally, the Boussinesq number must tend to zero as fast as the Mach number (which is a restriction of the vertical scale of the problem). In the conditions of the benchmark, the Boussinesq number is $5.7 \times 10^{-5}$ and is of the same order as the Mach number. Thus, the dimensionless parameters that define the problem are:

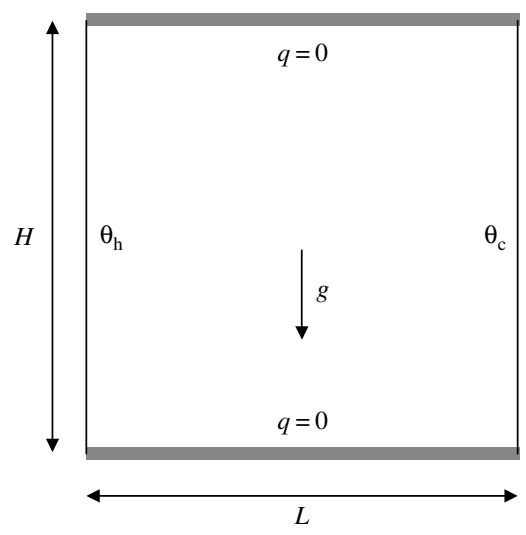

Finite element approximation

853

\section{Figure 1.} Geometry and boundary conditions of the natural convection in a cavity 


\section{$\mathrm{HFF}$}

$18,7 / 8$

$$
\begin{gathered}
\varepsilon=\frac{\vartheta_{h}-\vartheta_{c}}{\vartheta_{h}+\vartheta_{c}}, \quad \mathbf{A}=\frac{H}{L} \\
\operatorname{Pr}=\frac{c_{p} \mu}{k}, \quad R a=\operatorname{Pr} \frac{g L^{3}}{\nu^{2}} \varepsilon
\end{gathered}
$$

where $\mathrm{Pr}$ is the Prandtl number and $R a$ is the Rayleigh number.

Let us first present results that show the physical behavior of the problem (see Chenoweth and Paolucci (1986) for a full description of the physics of the problem). They have been obtained using a fine grid of $160 \times 160$ Ql (bilinear) elements refined towards the walls. The steady state problem has been directly solved (without time advancing) with a convergence tolerance for the nonlinear process of $10^{-8}$.

Figure 2 shows the streamline and temperature distribution obtained using the Boussinesq approximation for different Rayleigh numbers. For a Rayleigh number of $10^{3}$, there is only one vortex that covers the whole domain. When the Rayleigh number is increased, this vortex splits first in two and then the vortex distribution becomes more complex and the boundary layers on hot and cold walls become thinner.

When the low Mach number approximation is used, similar results are obtained, but some differences are found. For a fixed Rayleigh number of $10^{3}$, when the temperature increases, the central vortex moves to the right. This effect can be seen in Table I, where the position of the center of the vortex as a function of temperature difference is presented. For higher Rayleigh numbers the effect is similar: the flow is qualitatively similar although some differences appear when quantifying magnitudes.

An important difference between the Boussinesq and the low Mach number approximations is that the latter can describe phenomena related to the expansion of the flow. If a gas in a closed cavity is heated, basic thermodynamics implies that the pressure level should increase and this cannot be predicted using the Boussinesq approximation. In the case of the differentially heated cavity at $\varepsilon=0.6$, the mean thermodynamic pressure normalized using the initial pressure is 0.856 . This case was considered to test the mesh convergence of the proposed algorithm using graded meshes of $10 \times 10,20 \times 20,40 \times 40$ and $80 \times 80$ Ql elements. Table II presents the. thermodynamic pressure as a function of the mesh size. It is seen that the behavior is as expected and the results agree with those found in the literature. It is to be noted that the results presented in Heuveline (2003) correspond to a discretization with 855,556 degrees of freedom obtained by an adaptive procedure.

Let us now describe the nonlinear convergence of the iterative scheme when a direct steady state calculation is performed. A set of experiments were performed using the Boussinesq model for the different possible linearizations on a uniform mesh of $10 \times 10$ Q1 elements. Figure 3 shows the convergence of the algorithm for different Rayleigh numbers.

From these experiments, we conclude that the Newton scheme $\left(\lambda_{1,1}=1, \lambda_{1,3}=1\right.$, $\lambda_{3,1}=1$ ) is fastest, as expected, and the linearization $\lambda_{1,1}=0, \lambda_{1,3}=1, \lambda_{3,1}=0$ is the most robust for this example (in the sense that it converges for higher values of the Rayleigh number). When the buoyancy term is treated in a coupled way (taking $\lambda_{1,3}=1$ ) the convergence becomes monotone for $R a=10^{3}$ and $R a=10^{4}$ but it is also seen that what makes a big difference is to combine this treatment with a full Newton linearization of the convective term in the temperature equation (that is to take also $\lambda_{3,1}=1$ ). Let us stress that although a Picard type linearization could be more robust when 

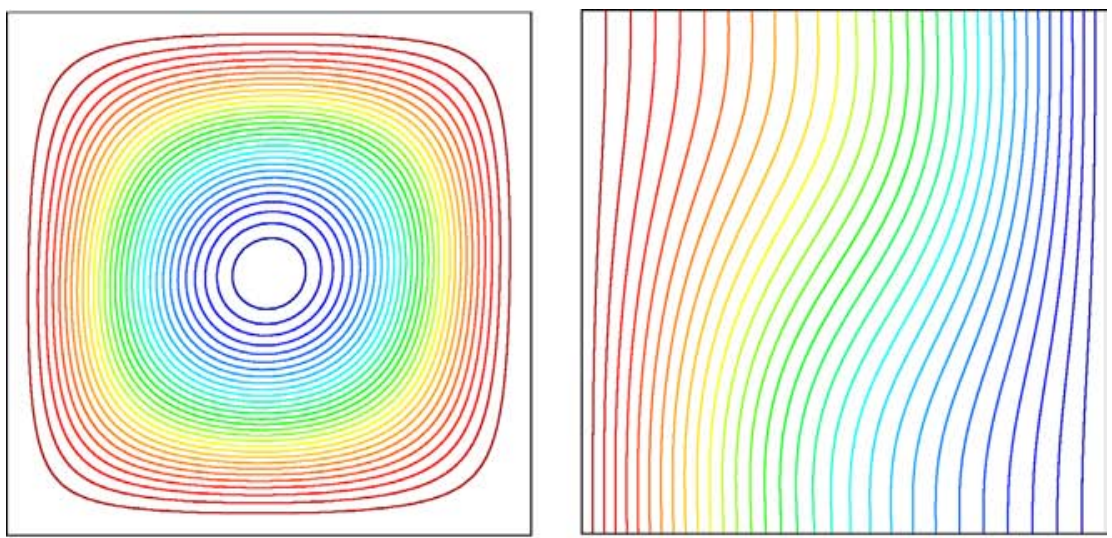

Finite element approximation
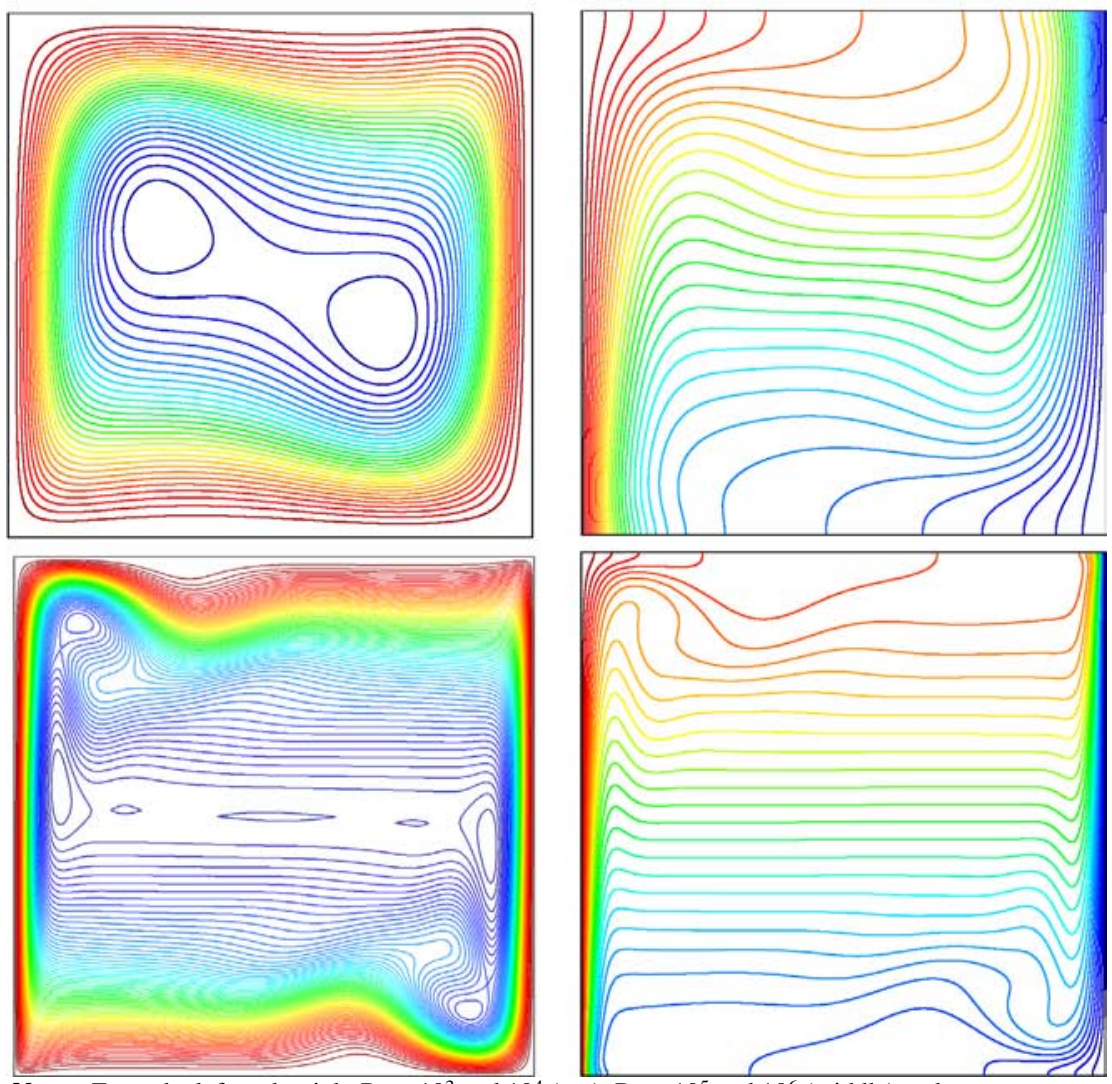

Notes: From the left to the right $\mathrm{Ra}=10^{3}$ and $10^{4}$ (top), $\mathrm{Ra}=10^{5}$ and $10^{6}$ (middle) and $\mathrm{Ra}=10^{7}$ and $10^{8}$ (bottom)

Figure 2.

Streamline distribution obtained using the Boussinesq approximation 
$\mathrm{HFF}$

$18,7 / 8$

856

Table I.

Evolution of the

$x$-coordinate of the central 0.2

vortex for $R a=10^{3}$ in $\quad 0.4$

terms of $\varepsilon$ incompressible Navier Stokes are considered (which is also observed here when comparing $\lambda_{1,1}=0, \lambda_{1,3}=1, \lambda_{3,1}=0$ and $\lambda_{1,1}=1, \lambda_{1,3}=1, \lambda_{3,1}=1$ ), a Newton type linearization of the velocity to the temperature coupling (in this case through the convective term in the temperature equation) is more robust.

Next, we performed a set of experiments to test the behavior of the line search process described in Section 4. For the full Newton linearization, we performed

\begin{tabular}{lc}
\hline$\varepsilon$ & $x$-coord \\
\hline 0.0 & 0.50 \\
0.2 & 0.54 \\
0.4 & 0.58 \\
0.6 & 0.63
\end{tabular}

$h$ convergence of the

\begin{tabular}{lcccc}
\hline$H$ & $R a=10^{3}$ & $R a=10^{4}$ & $R a=10^{5}$ & $R a=10^{6}$ \\
\hline 0.1000 & 0.8646791 & 0.8585644 & 0.8681438 & - \\
0.0500 & 0.8603283 & 0.8497187 & 0.8567441 & 0.8670059 \\
0.0250 & 0.8582884 & 0.8460002 & 0.8534048 & 0.8579742 \\
0.0125 & 0.8574004 & 0.8445817 & 0.8524585 & 0.8567541 \\
Reference (Heuveline, 2003) & - & - & - & 0.856337 \\
\hline
\end{tabular}

Figure 3.

Nonlinear convergence for the $10 \times 10$ uniform mesh
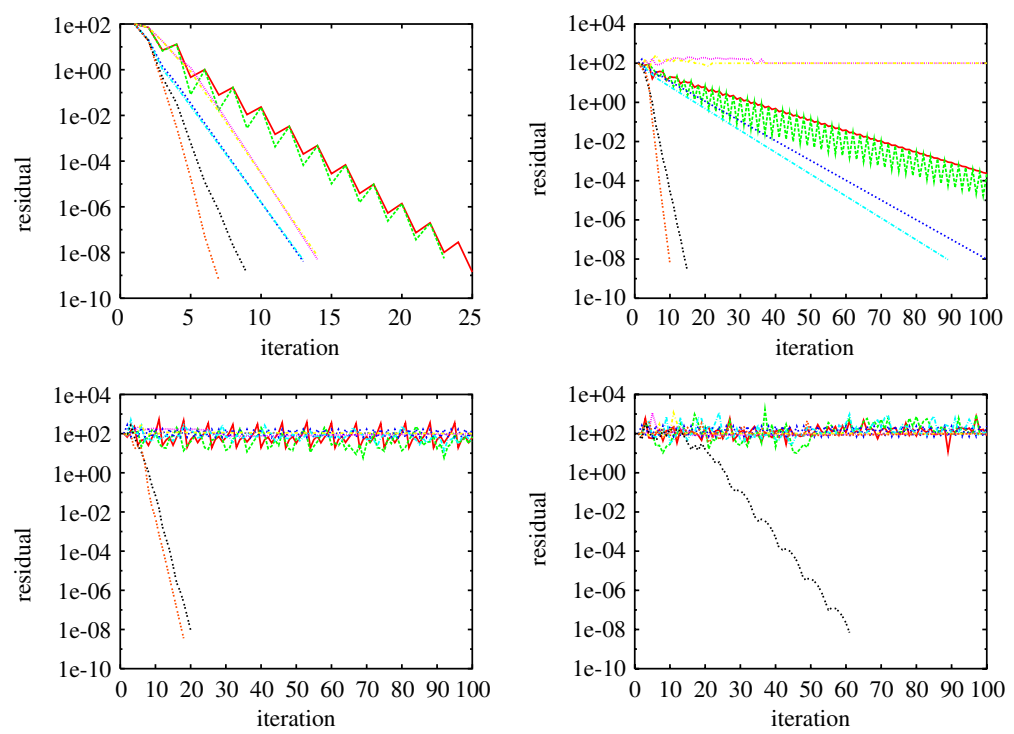

Notes: From left to right and top to bottom $\mathrm{Ra}=10^{3}, 10^{4}, 10^{5}$ and $10^{6}$. The curves corresponding to the linearization parameters and are given as follows:

$$
\begin{array}{rrrr}
000 & 010 & 011 \\
100 & 001 & 110 & 101
\end{array}
$$


computations for uniform meshes of $10 \times 10,20 \times 20,40 \times 40$ and $80 \times 80$ Q1 elements without a line search and using the Armijo rule described in Section 4 (the experiments using the Armijo rule were also run on a uniform mesh of $160 \times 160 \mathrm{Q} 1$ elements). Tables III and IV now the behavior of the iterative scheme by indicating the number of iterations needed when convergence is achieved.

Very similar results are obtained for the linearization that corresponds to $\lambda_{1,1}=0, \lambda_{1,3}=1, \lambda_{3,1}=1$. The conclusion to be drawn is that the use of the line search greatly improves the robustness of the iterative scheme. It is also to be mentioned that, although when a line search is not performed the linearization that corresponds to $\lambda_{1,1}=0, \lambda_{1,3}=1, \lambda_{3,1}=1$, converges for some cases where the full Newton does not, when using the Armijo rule both linearizations behave identically. Moreover, the calculations where the scheme fails to converge are on coarse meshes and convergence is achieved for finer meshes (especially when refined meshes are used). The linearization scheme for the low Mach number model has also been tested in detail and the same behavior was observed: the full Newton scheme together with the Armijo rule converges for almost any case. In Martinez and Gartling (2004), difficulties to obtain convergence when performing calculations using the low Mach number model have been reported even for a low Rayleigh number $\left(R a=10^{4}\right)$. In this reference, an ad hoc linearization of the system was performed to overcome this problem. We did not find this problems for low Rayleigh number. In Martinez and Gartling (2004), the problem is solved using a mixed finite element formulation, what could be the reason behind the difference in the behavior of the iterative algorithms.

We next consider the behavior of the line search process when a time dependent calculation is performed. A set of experiments were performed using the full Newton linearization and the Boussinesq model on a uniform mesh of $10 \times 10$ elements with and without line search. The number of iterations needed are shown in Tables V and VI.

\section{Finite element approximation}

857

\begin{tabular}{lcccccc}
\hline elem. & $R a=10^{3}$ & $R a=10^{4}$ & $R a=10^{5}$ & $R a=10^{6}$ & $R a=10^{7}$ & $R a=10^{8}$ \\
\hline 10 & 7 & 10 & 18 & - & - & - \\
20 & 6 & 9 & 14 & 20 & - & - \\
40 & 6 & 9 & 14 & - & - & - \\
80 & 6 & 8 & 13 & - & - & -
\end{tabular}

Note: The dash indicates divergence

Table III. Number of iterations for the linearization $\lambda_{1}=1, \lambda_{2}=1, \lambda_{3}=1$, without line search

\begin{tabular}{lcccccc}
\hline elem. & $R a=10^{3}$ & $R a=10^{4}$ & $R a=10^{5}$ & $R a=10^{6}$ & $R a=10^{7}$ & $R a=10^{8}$ \\
\hline 10 & 8 & 13 & 17 & 22 & 96 & 64 \\
20 & 8 & 12 & 13 & 17 & 34 & 162 \\
40 & 8 & 12 & 13 & 14 & 17 & $*$ \\
80 & 9 & 12 & 13 & 14 & 16 & 24 \\
160 & 7 & 13 & 14 & 16 & 17 & 26
\end{tabular}

Note: The star indicates lack of convergence

Table IV. Number of iterations for the linearization $\lambda_{1}=1, \lambda_{2}=1, \lambda_{3}=1$, using the Armijo rule 
$\mathrm{HFF}$

$18,7 / 8$

858

Table V.

Number of iterations at the first time step for the linearization $\lambda_{1}=1, \lambda_{2}=1, \lambda_{3}=1$, without line search
As it could be expected, and these experiments confirm, less-nonlinear iterations are required to achieve convergence when the time step is reduced. These experiments also show that still the line search algorithm is important when time steps are big and the nonlinearity is important. When the time step is reduced, convergence is achieved without the need of relaxation.

We next consider the nonlinear convergence of the iterative scheme when a time dependent calculation is performed. A set of experiments were performed using the linearization that corresponds to $\left(\lambda_{1,1}=0, \lambda_{1,3}=0, \lambda_{3,1}=0\right)$ and the Boussinesq model on a uniform mesh of $10 \times 10$ Ql elements (Table VII).

The main conclusion of these experiments is that when the time step is small, the number of iterations required by a Picard type linearization $\left(\lambda_{1,1}=0, \lambda_{1,3}=0, \lambda_{3,1}=0\right)$ or those required by a Newton type one $\left(\lambda_{1,1}=1, \lambda_{1,3}=1, \lambda_{3,1}=1\right)$ are similar. In this cases, the Picard type linearization is preferred because it allows a splitting of the algebraic problem into a mechanical problem and a thermal problem. Note that this is interesting because it permits to modify an incompressible code to take thermal coupling into account and because it permits to reduce memory requirements storing smaller

\begin{tabular}{|c|c|c|c|c|c|c|}
\hline$\delta t$ & $R a=10^{3}$ & $R a=10^{4}$ & $R a=10^{5}$ & $R a=10^{6}$ & $R a=10^{7}$ & $R a=10^{8}$ \\
\hline
\end{tabular}

\begin{tabular}{llrrrrr}
\hline $10^{0}$ & 7 & 10 & 18 & 35 & - & - \\
$10^{-1}$ & 6 & 9 & 16 & 22 & - & - \\
$10^{-2}$ & 5 & 8 & 12 & 20 & - & - \\
$10^{-3}$ & 5 & 6 & 11 & 23 & 33 & - \\
$10^{-4}$ & 4 & 5 & 7 & 13 & 53 & 204
\end{tabular}

Note: The dash indicates divergence

\section{Table VI.}

Number of iterations at the first time step for the linearization $\lambda_{1}=1, \lambda_{2}=1, \lambda_{3}=1$, using the Armijo rule

\begin{tabular}{lcccccc}
\hline St & $R a=10^{3}$ & $R a=10^{4}$ & $R a=10^{5}$ & $R a=10^{6}$ & $R a=10^{7}$ & $R a=10^{8}$ \\
\hline $10^{0}$ & 7 & 13 & 17 & 22 & 118 & $*$ \\
$10^{-1}$ & 6 & 12 & 16 & 22 & $*$ & $*$ \\
$10^{-2}$ & 5 & 8 & 13 & 22 & 45 & $*$ \\
$10^{-3}$ & 5 & 6 & 11 & 23 & 40 & 60 \\
$10^{-4}$ & 4 & 5 & 7 & 13 & 53 & 52
\end{tabular}

Note: The star indicates lack of convergence

Table VII.

Number of iterations at the first time step for the linearization $\lambda_{1}=0, \lambda_{2}=0, \lambda_{3}=0$, without line search

\begin{tabular}{lcccccc}
\hline$\delta t$ & $R a=10^{3}$ & $R a=10^{4}$ & $R a=10^{5}$ & $R a=10^{6}$ & $R a=10^{7}$ & $R a=10^{8}$ \\
\hline $10^{0}$ & 23 & 166 & - & - & - & - \\
$10^{-1}$ & 17 & 85 & - & - & - & - \\
$10^{-2}$ & 9 & 14 & 39 & $*$ & 200 & - \\
$10^{-3}$ & 6 & 8 & 13 & 32 & $*$ \\
$10^{-4}$ & 5 & 6 & 7 & 13 & 48 & $*$
\end{tabular}

Note: The dash indicates lack of convergence and the star lack of convergence on the first steps 
matrices (but note also that the time required to solve the linear system will be the same). This leads to three types of iterative coupling: the one that corresponds to $\lambda_{1,1}=0, \lambda_{1,3}=0, \lambda_{3,1}=0$, that permits the parallel solution of the mechanical and the thermal problem, and those that correspond to $\lambda_{1,1}=0, \lambda_{1,3}=0, \lambda_{3,1}=1$ and $\lambda_{1,1}=0, \lambda_{1,3}=1, \lambda_{3,1}=0$ which result in a Gauss Seidel type scheme.

The difference between the last two of them is which problem is solved first. It can be observed in Figure 3 that the linearization that corresponds to $\lambda_{1,1}=0, \lambda_{1,3}=1, \lambda_{3,1}=0$, that is the one in which the thermal problem is solved first, is more robust than the one that corresponds to $\lambda_{1.1}=0, \lambda_{1.3}=0, \lambda_{3,1}=1$, that is the one in which the mechanical problem is solved first. Respect to this point, let us also mention that in the case of the low Mach number system, it is quite important to solve for the temperature first because of the term ${ }_{t} \rho$ in the continuity equation. If the mechanical problem is solved first, in the first iteration of the time step, the approximation to ${ }_{t} \rho$ is zero as the initial guess is the temperature at the previous step and this needs to be corrected by the iterative coupling. This effect has been observed while solving the problem presented in the following section (Table VIII).

\subsection{Time dependent heated channel}

The problem studied here, shown in Figure 4, is a channel whose length $(L)$ is 5 times it; height $(H)$.

The inlet boundary conditions are given by a Poiseuille velocity profile and uniform temperature. A non slip condition is prescribed on the upper and lower walls. Zero heat flux is prescribed on the upper wall and on the part of the lower wall where temperature is not pre-scribed, as shown in Figure 4. A time dependent temperature is prescribed on a part of the lower wall of length $H / 2$ located at a distance $H / 2$ from the inlet. The prescribed (dimensionless) temperature rises from 1 at time $t=0$ to 1.5 at time $t=0.01$ and remains constant after that. The dimensionless parameters of the problem are:

$$
\mathbf{R}=\frac{\rho U L}{\mu}=10, \quad \operatorname{Pr}=\frac{c_{p} \mu}{k}=1, \quad \varepsilon=\frac{\Delta T}{T_{0}}=0.5, \quad \operatorname{Ra}=\operatorname{Pr} \frac{g L^{3}}{\nu^{2}} \varepsilon=5 \times 10^{4}
$$

\begin{tabular}{lcccccc}
\hline$\delta t$ & $R a=10^{3}$ & $R a=10^{4}$ & $R a=10^{5}$ & $R a=10^{6}$ & $R a=10^{7}$ & $R a=10^{8}$ \\
\hline $10^{0}$ & 14 & 132 & - & - & - & - \\
$10^{-1}$ & 1 & 47 & - & - & - & - \\
$10^{-2}$ & 8 & 11 & 25 & - & - & - \\
$10^{-3}$ & 7 & 8 & 12 & 33 & - & - \\
$10^{-4}$ & 5 & 5 & 7 & 13 & 79 & $*$
\end{tabular}

Note: The dash indicates lack of convergence and the star lack of convergence on the first steps

\section{Finite element approximation}

859

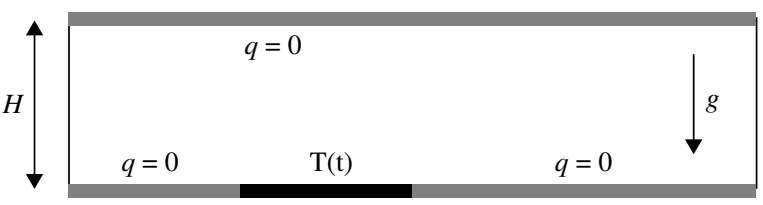

Figure 4. Geometry and boundary conditions of the time dependent heated channel (not to scale)

Number of iterations at the first time step for the

linearization

$\lambda_{1}=0, \lambda_{2}=0, \lambda_{3}=1$ without line search 


\section{$\mathrm{HFF}$}

$18,7 / 8$

\section{0}

The initial conditions axe a Poiseuille velocity profile and a constant temperature on the whole domain. When the flow starts to heat (near the zone where the temperature is imposed), it goes up by buoyancy forces giving rise to two vortices, one before and the other after the heating zone. This is shown in Figure 5, where streamlines and temperature distribution obtained using the Boussinesq approximation are presented for different (early) times.

After this initial transient, the flow after the heating zone gradually rises its temperature and the second vortex (the one located after the heating zone) gradually disappears. The final steady state is reached around $t=12$. This behavior is also observed when the low Mach number approximation is used.

The first point we want to illustrate here is the influence of the output boundary condition. One possibility is to consider simply:

$$
t \cdot n=0
$$

but it seems to be better to consider an "atmospheric stress condition" as the one suggested in Martinez and Gartling (2004), given by:

$$
t \cdot n=t_{x}=-\rho|g| y
$$

The final steady state obtained using these conditions is shown in Figure 6.

\section{Tunnel fire}

This problem is a simplified simulation of what would occur at the beginning of a fire in a tunnel. A fire is a complex phenomenon whose detailed simulation involves many different, aspects that are beyond the scope of this work. The simplified model considers the fire as a source of heat, without taking into account the exact reactive mechanism that would imply a precise knowledge of the chemical components of the fuel and the solution of a complete reactive problem. However, one important aspect of the problem is that the high-temperature differences that are present do not permit the
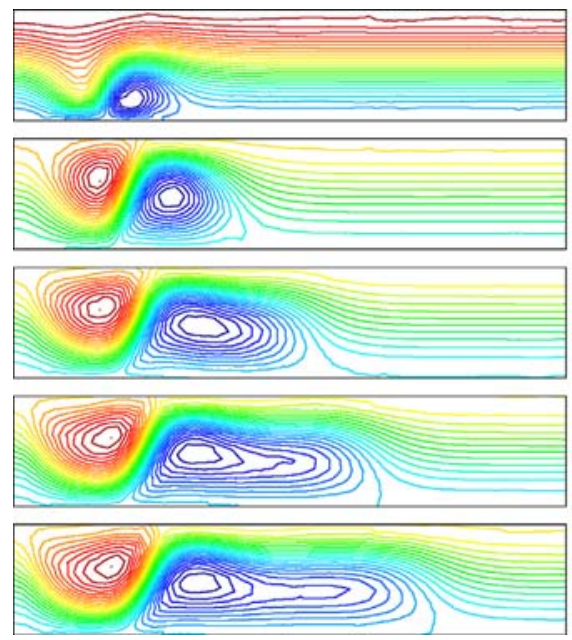

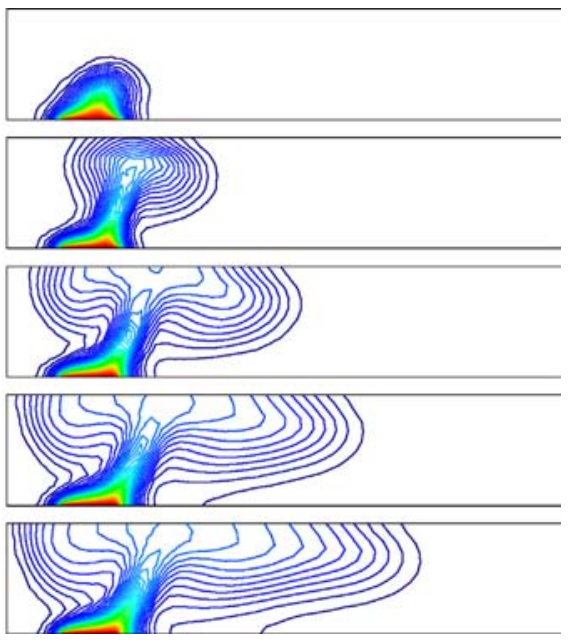

Figure 5.

Streamlines and temperature distribution obtained using the Boussinesq approximation at times $t=0.2, t=0.4$, $t=0.6, t=0.8$ and $t=1.0$ 
simulation of the problem using the Boussinesq approximation and makes the use of the low Mach number model mandatory.

Two simulations were carried out considering heat sources of 0.8 and $4 \mathrm{MW}$, which correspond to a small size fire (a car for example) distributed in a volume of $8 \mathrm{~m}^{3}$. Based on experimental results, a typical wind in a tunnel in absence of fire has a velocity of about $0.5 \mathrm{~m} / \mathrm{s}$ (which is used to define the time scale taking $\mathbf{S}=1$ ). Then, the dimensionless numbers are:

$$
\mathbf{S}=1, \quad \mathbf{M} \sim 1.7 \times 10^{-3}, \quad \mathbf{R}=1.6 \times 10^{5}, \quad P r=0.71, \quad \mathbf{F}=7.1 \times 10^{-2}, H=2.8-14.1
$$

As the Mach number is small the use of the low Mach number approximation is justified. This is not the case for the Boussinesq approximation. Although the squared Froude number $\left(\sim 5 \times 10^{-3}\right)$ can be considered of the order $\mathbf{M}$, the heat release number is not small. In other words, the height of the tunnel can be considered small (with respect to the variation of thermodynamic fields in the atmosphere) but temperature differences are not small. It is also to be noted that the high-Reynolds number of the problem implies the need, of taking turbulence into account what we do introducing a Smagorinsky (Sagaut, 2001) eddy viscosity defined by:

$$
\mu^{t}=\rho c_{s} \Delta^{2}\left[\varepsilon^{\prime}(u): \varepsilon^{\prime}(u)\right]^{1 / 2}
$$

where $c_{s}$ is an empirical constant and $\Delta$ a characteristic length usually taken as the mesh size. A subgrid thermal conductivity is also added. It is defined in terms of the subgrid viscosity as:

$$
k^{t}=\frac{\mu^{t} c_{p}}{P r^{t}}
$$

where $\mathrm{Pr}^{\mathrm{t}}$ is the turbulent Prandtl number that is assumed to be constant (and taken to be 0.5). A preliminary calculation was performed to reproduce the initial state of a wind flowing through the tunnel which was obtained applying a pressure difference on the tunnel portals. On the tunnel walls, Neumann boundary conditions based on universal profiles were applied (wall laws). They can be written as:

$$
t=-\frac{\rho u_{*}^{2}}{\|u\|} u
$$

where $u^{*}$ (the friction velocity) is the solution of an equation of the form:
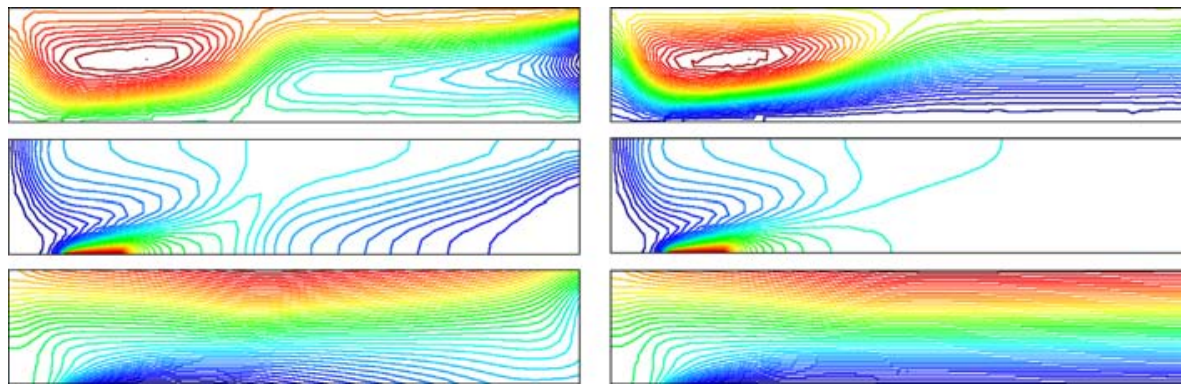

Finite element approximation

861 
$\mathrm{HFF}$

$18,7 / 8$

862

$$
u^{+}:=\frac{\|u\|}{u_{*}}=f\left(y^{+}\right)= \begin{cases}y^{+} & \text {if } y^{+}<11.6 \\ 2.5 \log \left(y^{+}\right)+5.5 & \text { if } y^{+} \geq 11.6\end{cases}
$$

and $y^{+}=y u_{*} / \nu, y$, being the distance normal to the wall. Let us remark that this boundary condition introduces yet another important nonlinearity to the system (when a fully developed isothermal flow is considered it is the dominant one as the convective term almost vanishes). The common practice is to evaluate the traction using the previous iterate of the velocity, but we found better to send it to the left hand side. At the nonlinear iteration $i$, the friction velocity is found solving:

$$
\frac{\left\|u^{i-1}\right\|}{u_{*}}=f\left(\frac{y u *}{\nu}\right)
$$

and the traction calculated as:

$$
t^{i}=-\frac{\rho u_{*}^{2}}{\left\|u^{i-1}\right\|} u^{i}
$$

How important the gain is depends on the time step size, as shown in Figure 7, where the convergence evolution of the velocity in a plane channel flow for two different time steps is displayed.

Boundary conditions for temperature were defined to reproduce the real situation as close as possible. On the tunnel walls, a Robin type condition was applied using a convection coefficient $\alpha_{\text {conc }}$ suggested by laboratory experiments and the temperature on the concrete walls was fixed to $\vartheta_{\text {conc }}$, so that this condition reads:

$$
\left(k+k^{t}\right) \frac{\partial \vartheta}{\partial n}+\alpha_{\text {conc }}\left(\vartheta-\vartheta_{\text {conc }}\right)=0
$$

On the entrance and exit of the tunnel, homogeneous Neumann boundary conditions were considered.

The physics of the flow is quite complex and the temporal evolution is chaotic. When the heating starts, strong buoyancy forces determine the formation of a plume and recirculation zones that now, in contrast to the previous example, are fully tridimensional and of complex structure. In Figure 8, the velocity fields for the two heat sources considered at 3 min after the starting of the heating are shown, and in Figure 9 the corresponding temperature fields arc shown. Both figures show a detail of the fire

Figure 7.

Velocity norms as a function of the iteration for time step $\delta t=1$ (left) and $\delta t=5$ (right) in a plane channel flow
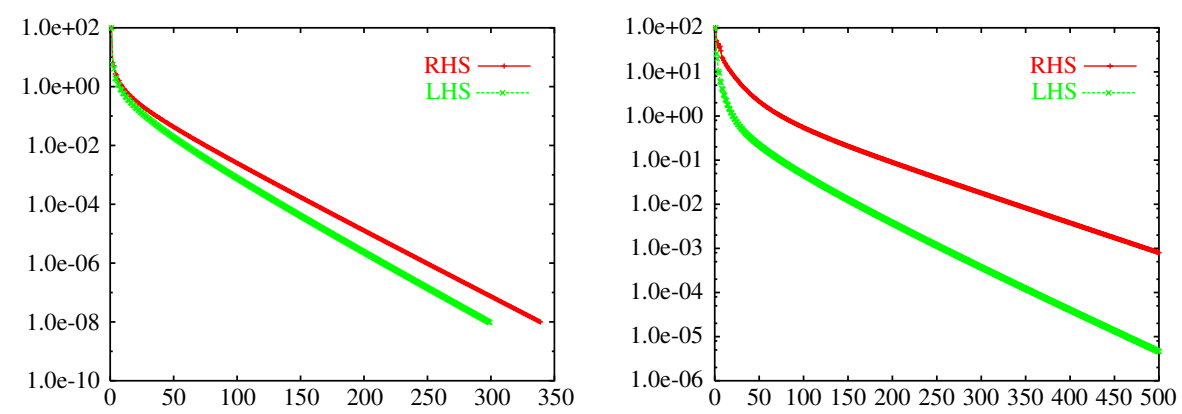
zone introducing cutting planes that intersect the fire zone. The heat source generates the plume that can be clearly shown in Figure 8 where an expansion of the flow is also apparent. This expansion is better shown in Figure 10 where contour lines of divergence of the velocity are shown. They have been obtained by projecting velocity gradients on the finite element space.

Let us finally make some comments on numerical aspects of the simulations carried out. As relatively small time steps have been used, a block iterative scheme for the momentum-temperature coupling has been used, which converged in 4 or 5 iterations. The linear system has been solved using a GMRES method (Saad, 1996) preconditioned using an ILUT (nfill,thres) strategy described in Saad (1994), where nfill denotes the level of filling and acts as a memory limiter and thres is a threshold for the choice of filling elements and acts as a cpu time limiter. Several combinations of nfill and thres have been tested for this problem and it was observed that, as expected, increasing the filling and reducing the threshold reduces the number of GMRES iterations needed to achieve convergence. The optimal compromise depends on the particular problem
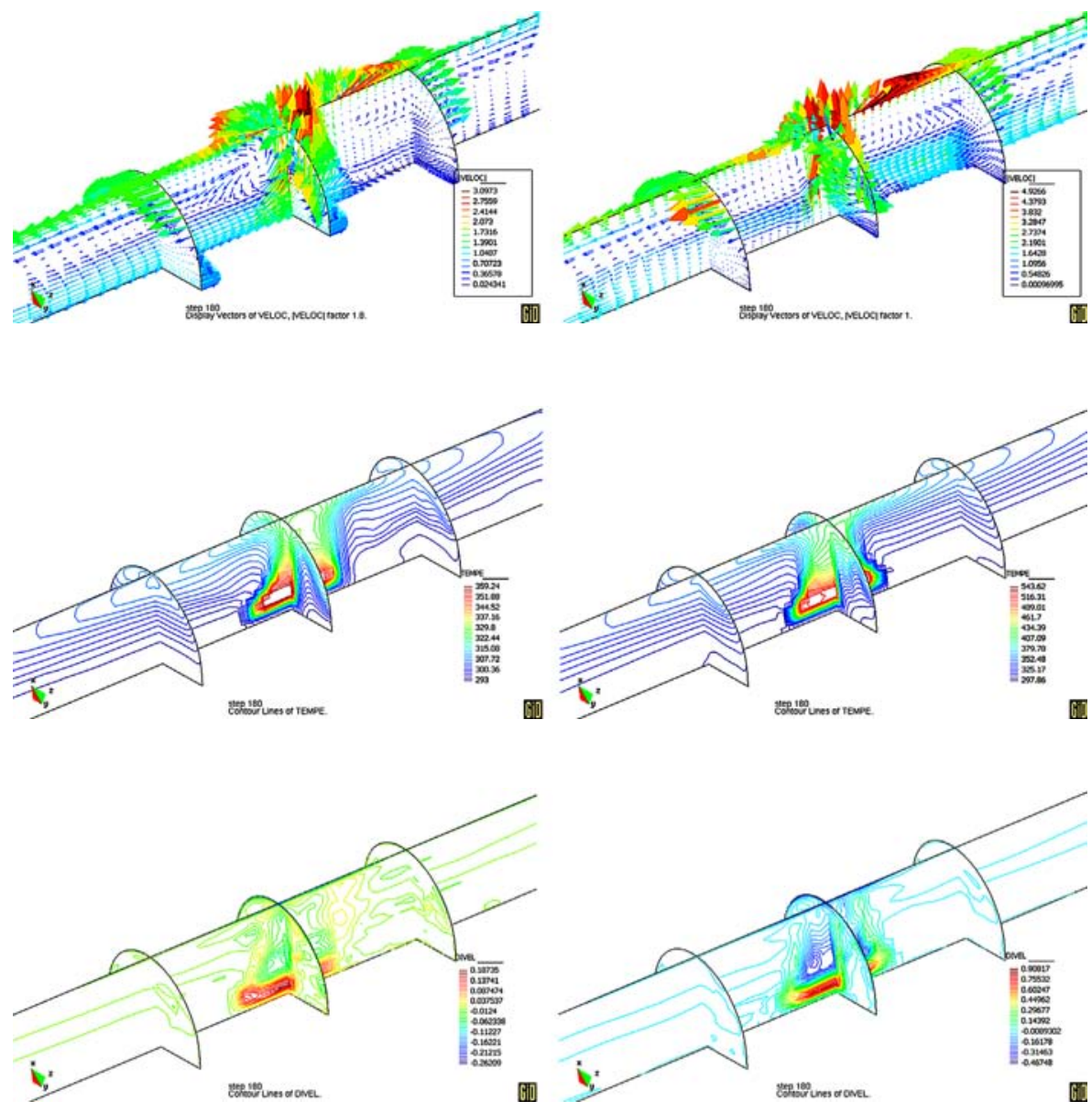

Finite element approximation

863

Figure 8.

Velocity fields at $t=180 \mathrm{~s}$

for $Q=0.1 \mathrm{mw} / \mathrm{m}^{3}$ (left) and $Q=0.5 \mathrm{mw} / \mathrm{m}^{3}$ (right)

Figure 10.

Divergence of the velocity fields at $t=180 \mathrm{~s}$ for $Q=0.1 \mathrm{mw} / \mathrm{m}^{3}$ (left) and $Q=0.5 \mathrm{mw} / \mathrm{m}^{3}$ (right)
Figure 9. Temperature fields at $t=180 \mathrm{~s}$ for $Q=0.1 \mathrm{mw} / \mathrm{m}^{3}$ (left) and $Q=0.5 \mathrm{mw} / \mathrm{m}^{3}$ (right) 


\section{$\mathrm{HFF}$}

$18,7 / 8$

864 considered (including mesh size, initial and boundary conditions, etc.). Let us only point out that this method is more efficient for higher time steps. This is shown in Figure 11, where the residuals after 100 iterations of GMRES as a function of the nfill parameter are shown for a threshold of $10^{-2}$.

\section{Conclusions}

The zero Mach number model and the Boussinesq approximation have been considered to solve low speed thermally coupled flows. Both describe the basic mechanism of thermal coupling which is due to the dependence of the density on the temperature: when a fluid element is heated, it expands and moves up. However, they differ in the way they take into account the compressibility of the medium. While in the Boussinesq approximation, the flow is incompressible, in the zero Mach number model the density distribution is predicted and the velocity field is affected by expansions or contractions due to heating. They also have different ranges of applicability: while the low Mach number approximation only requires a small Mach number, the Boussinesq model requires also a small Froude number and small heat sources (Principe and Codina, 2008).

The numerical formulation of these problems based on the subgrid scale approach has proved to be effective. The well-known instabilities present in convection dominated problems and those due to the equal order interpolation of all the unknowns have been circumvented. In addition, a nonlinear transient stabilization technique which is based on allowing the subscales to vary in time and applying the subgrid decomposition on the nonlinear terms leads to important improvements on the scheme although numerical results have not been presented here (Codina et al., 2007; Codina and Principe, 2007).

We have implemented the discrete approximation to these problems as systems of second order equations. Different linearizations have been considered for the solution of the algebraic nonlinear problem. Using the well-known example of the flow in a differentially heated cavity, we have shown that fully coupled schemes, in which a Newton type linearization is performed, are the best option for the solution of stationary problems or when the time step is large. In this case, a line search strategy is very important to enhance the robustness of the scheme. Its cost is higher to that of

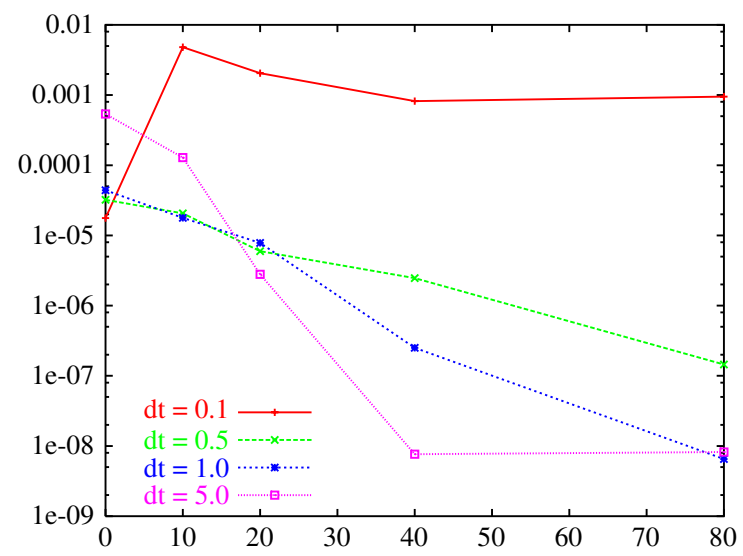

Figure 11.

GMRES convergence for different time step sizes 
forming the system of equations and for this reason it is not convenient when small time steps are considered. In this case, there is also a small difference between the full Newton linearization and the staggered approach in which the problem is split into a mechanical problem and a thermal one. It has also been shown that in this case it is better to solve the thermal problem first. A fractional step scheme, splitting also momentum and continuity equations, could be considered in this case, but this is a point that needs further research.

\section{References}

Batchelor, G.K. (1967), An Introduction to Fluid Dynamics, Cambridge University Press, Cambridge.

Bois, P.A. (1976), "Propagation linéare et non linéaire d’ondes atmosphériques", Journal de Mécanique, Vol. 15 No. 5, pp. 781-811.

Bois, P.A. (1991), "Asymptotic aspects of the Boussinesq approximation for gases and liquids", Geophysics and Astrophysics Fluid Dynamics, Vol. 58, pp. 45-55.

Boussinesq, J. (1903), Théorie analytique de la chaleur, Vol. 2, Gauthier-Villars, Paris (Reproduction Bibliothéque Nationale de France, 1995).

Buckingham, E. (1914), "On physically similar systems; illustrations of the use of dimensional equations”, Physical Review Series II, Vol. 4 No. 4, pp. 345-76.

Chenoweth, D.R. and Paolucci, S. (1986), "Natural convection in an enclosed vertical air layer with large horizontal temperature differences", Journal of Fluid Mechanics, Vol. 169, pp. 173-210.

Codina, R. (1998), "Comparison of some finite element methods for solving the diffusion-convection-reaction equation", Computer Methods in Applied Mechanics and Engineering, Vol. 156, pp. 185-210.

Codina, R. (2000a), "On stabilized finite element methods for linear systems of convection-diffusion-reaction equations", Computer Methods in Applied Mechanics and Engineering, Vol. 188 Nos 1/3, pp. 61-82.

Codina, R. (2000b), "Stabilization of incompressibility and convection through orthogonal subscales in finite element methods", Computer Methods in Applied Mechanics and Engineering, Vol. 190 Nos 13/14, pp. 1579-99.

Codina, R. (2001), "A stabilized finite element method for generalized stationary incompressible flows", Computer Methods in Applied Mechanics and Engineering, Vol. 190 Nos 20/21, pp. 2681-706.

Codina, R. (2002), "Stabilized finite element approximation of transient incompressible flows using orthogonal subscales", Computer Methods in Applied Mechanics and Engineering, Vol. 191 Nos 39/40, pp. 4295-321.

Codina, R. and Principe, J. (2007), "Dynamic subscales in the finite element approximation of thermally coupled incompressible flows", International Journal for Numerical Methods in Fluids, Vol. 54 Nos 6/8, pp. 707-30.

Codina, R., Principe, J., Guasch, O. and Badia, S. (2007), "Time dependent subscales in the stabilized finite element approximation of incompressible flow problems", Computer Methods in Applied Mechanics and Engineering, Vol. 196 Nos 21/24, pp. 2413-30.

Davis, G.D.V. and Jones, I. (1983), "Natural convection in a square cavity: a comparison exercise", International Journal for Numerical Methods in Fluids, Vol. 3, pp. 227-48.

\section{Finite element} approximation

865 


\section{HFF}

$18,7 / 8$

\section{6}

Dennis, J. and Schnabel, R. (1983), Numerical Methods for Unconstrained Optimization and Non-linear Equations, 1st ed., Prentice-Hall series in Computational Mathematics, Prentice-Hall, Englewood Cliffs, NJ.

Franca, L.P. and Farhat, C. (1995), "Bubble functions prompt unusual stabilized finite element methods", Computer Methods in Applied Mechanics and Engineering, Vol. 123, pp. 299-308.

Franca, L.P., Frey, S. and Hughes, T. (1992), "Stabilized finite element methods: I. application to the advective-diffusive model”, Computer Methods in Applied Mechanics and Engineering, Vol. 95, pp. 253-76.

Gray, D.D. and Giorgini, A. (1976), "The validity of the Boussinesq approximation for liquids and gases", International Journal of Heat and Mass Transfer, Vol. 19 No. 5, pp. 545-51.

Heuveline, V. (2003), "On higher-order mixed fem for low Mach number flows: application to a natural convection benchmark problem", International Journal for Numerical Methods in Fluids, Vol. 41, pp. 1339-56.

Hughes, T.J.R. (1995), "Multiscale phenomena, Green's functions, the Dirichlet-to-Neuman formulation, subgrid scale models, bubbles and the origins of stabilized methods", Computer Methods in Applied Mechanics and Engineering, Vol. 127, pp. 387-401.

Hughes, T.J.R., Franca, L.P. and Balestra, M. (1986), “A new finite element formulation for computational fluid dynamics: V. circumventing the Babuska-Brezzi condition: a stable Petrov-Galerkin formulation of the stokes problem accommodating equal-order interpolations", Computer Methods in Applied Mechanics and Engineering, Vol. 59 No. 1, pp. 85-99.

Jensen, K., Einset, E. and Fotiadis, D. (1991), "Flow phenomena in chemical vapor deposition of thin films", Annual Review of Fluid Mechanics, Vol. 23, pp. 197-233.

Kelley, C.T. (1999), Iterative Methods for Optimization, SIAM, Philadelphia, PA.

Kevorkian, J. and Cole, J.D. (1981), Perturbation Methods in Applied Mathematics. Vol. 34 of Applied Mathematical Sciences, Vol. 34, Springer, New York, NY, (see also Kevorkian on bib.upc 1996 and 2000 books).

Kolmogorov, A.N. and Fomin, S.V. (1999), Elements of the Theory of Functions and Functional Analysis, Dover Publications, New York, NY.

Le Quéré, P. and Paillére, P. (2000), "Modelling and simulation of natural flows with large temperature differences: a benchmark problem for low Mach number solvers", paper presented at Workshop/12th CFD Seminar, CEA Saclay, Saclay, pp. 14-22.

Lions, P.L. (1996), Mathematical Topics in Fluid Dynamics, Incompressible Models, Vol. 1, Oxford University Press, Oxford.

Majda, A. and Sethian, J. (1985), "The derivation and numerical solution of the equations for zero Mach number combustion”, Combustion Science and Technology, Vol. 42, pp. 185-205.

Martinez, M. and Gartling, D. (2004), "A finite element method for low-speed compressible flows", Computer Methods in Applied Mechanics and Engineering, Vol. 193 Nos 21/22, pp. 1959-79.

Mihaljan, J.M. (1962), "A rigorous exposition of the Boussinesq approximations applicable to a thin layer of fluid”, Astrophysics Journal, Vol. 136 No. 3, pp. 1126-33.

Nicolas, X. (2002), "Revue bibliographique sur les ecoulements de Poiseuille-Rayleigh-Benard: ecoulements de convection mixte en conduites rectangulaires horizontales chauffees par le bas" ("Bibliographical review on the Poiseuille-Rayleigh-Benard flows: the mixed convection flows in horizontal rectangular ducts heated from below"), International Journal of Thermal Sciences, Vol. 41 No. 10, pp. 961-1016. 
Paolucci, S. (1982), "On the filtering of sound from the Navier-Stokes equations", Technical Report 82-8257, Sandia National Laboratories.

Perez Cordon, R. and Velarde, M.G. (1975), "On the (non-linear) foundations of Boussinesq approximation applicable to a thin-layer of fluid", Journal de physique, Vol. 36 No. 7, pp. 591-601.

Press, W., Flannery, B., Teukolsky, S. and Vetterling, W. (1992), Numerical Recipes: The Art of Scientific Computing, 2nd ed., Cambridge University Press, Cambridge.

Principe, J. and Codina, R. (2008), "On the low Mach number and the boussinesq approximations", Technical Report, CIMNE, Barcelona.

Rehm, R.G. and Baum, H.R. (1978), "The equations of motion for thermally driven bouyant flows", Journal of Research of the National Bureau of Standards, Vol. 83 No. 3, pp. 297-308.

Saad, Y. (1994), "Ilut: a dual threshold incomplete ilu factorization", Numerical Linear Algebra with Applications, Vol. 1, pp. 387-402.

Saad, Y. (1996), Iterative Methods for Saprse Linear Systems, 1st ed., PWS Publishing Company, Boston, MA.

Sagaut, P. (2001), Large Eddy Simulation for Incompressible Flows. Scientific Computing, Springer, Berlin.

Spiegel, E.A. and Veronis, G. (1960), "On the Boussinesq approximation for a compressible fluid”, Astrophysics Journal, Vol. 131, pp. 442-7.

Zeytounian, R.K. (1974), "A rigorous derivation of the equations of compressible viscous fluid motion with gravity at low Mach number”, Archiwum Mechaniki Stosowanej, Vol. 26, pp. 499-509.

Zeytounian, R.K. (1990), Asymptotic Modelling of Atmospheric Flows, Springer, Heidelberg.

Zeytounian, R.K. (2003), "Joseph Boussinesq and his approximation: a contemporary view", Comptes Rendus Mecanique, Vol. 331 No. 8, pp. 575-86.

\section{Corresponding author}

Javier Principe can be contacted at: principe@cimne.upc.edu

\section{Finite element approximation}

To purchase reprints of this article please e-mail: reprints@emeraldinsight.com Or visit our web site for further details: www.emeraldinsight.com/reprints 